\title{
Aperçu sur le commerce international et les prix domestiques dans le Bas-Canada (1793-1812)
}

\section{Gilles Paquet et Jean-Pierre Wallot}

Volume 21, numéro 3, décembre 1967

URI : https://id.erudit.org/iderudit/302692ar

DOI : https://doi.org/10.7202/302692ar

Aller au sommaire du numéro

Éditeur(s)

Institut d'histoire de l'Amérique française

ISSN

0035-2357 (imprimé)

1492-1383 (numérique)

Découvrir la revue

Citer cet article

Paquet, G. \& Wallot, J.-P. (1967). Aperçu sur le commerce international et les prix domestiques dans le Bas-Canada (1793-1812). Revue d'histoire de l'Amérique française, 21(3), 447-473. https://doi.org/10.7202/302692ar d'utilisation que vous pouvez consulter en ligne. 


\section{APERÇU SUR LE COMMERCE INTERNATIONAL ET LES PRIX DOMESTIQUES DANS LE BAS-CANADA (1793-1812)*}

Dans l'avant-propos de son "essai d'histoire quantitative et globale", Fernand Ouellet ${ }^{1}$ souligne le "renouveau amorcé dans la recherche historique" au Canada français, depuis environ deux décennies. De fait, la fresque aux traits accusés qu'il brosse du siècle qui suivit la Conquête, au Québec, veut s'inscrire dans "cet effort de renouvellement". Or, cette rénovation comporte deux composantes. D'abord, depuis Lucien Febvre et Marc Bloch, "l'explication historique a tendu à se socialiser" 2. Ce sont les groupes, plus ou moins organisés, non les individus qui intéressent la nouvelle histoire sociale. En outre, les méthodes d'approche ont aussi changé: des historiens ont admis, puis préféré "le recours nécessaire à la mesure et au quantitatif" 3 . L'histoire "sérielle" et l'axe "espace-temps" se sont imposés." Sous ces deux aspects - considération des groupes, recours au quantitatif -, Ouellet se greffe sur l'école française et son œuvre s'avère un solide "point de départ" en histoire canadienne.

Certes, une synthèse aussi vaste, qui embrasse près de cent ans, ne peut s'attarder aux détails ni examiner à fond les problèmes locaux ou particuliers. Cette esquisse à gros traits appelle donc un grand nombre de monographies qui viendront l'approfondir et la nuancer. En ce qui a trait à l'aspect quan-

* Nous remercions nos assistants de recherches, MM. Claude Ménard et Peter Sadlier-Brown, ainsi que le Musée national, l'Université Carleton (Ottawa) et l'Université de N[ontréal pour leur appui financier.

1 Fernand Ouellet, Histoire économique et sociale du Québec, 17601850 (Montréal, 1966).

2 Ernest Labrousse, "Introduction" in L'Histoire sociale - Sources et méthodes (ouvrage en collaboration, Paris, 1967), 2. 11.

3 Albert Soboul, "Description et mesure en histoire sociale", in ibid.,

4 Voir la courte note de Pierre Chaunu, "L'Histoire sérielle", dans La Revue d'Histoire de l'Amérique française, 21 (septembre 1967): 182-183. 
titatif, par exemple, Ouellet (et avec lui, Jean Hamelin) fonde son analyse sur une structure sérielle qui en est le fil conducteur. Mais les faiblesses et les lacunes de ces séries statistiques, ${ }^{5}$ l'absence d'analyse proprement économique et de modèle formel ainsi que l'insistance sur un exposé trop fortement conjoncturel font, qu'en fait, cette histoire "globale" ne parvient pas à digérer et à intégrer suffisamment le matériel quantitatif, non plus qu'à assumer toutes les dimensions du réel. En conséquence, l'œuvre de Ouellet n'est pas une histoire globale au sens de Braudel, ou une histoire quantitative au sens de Marczewski. ${ }^{6}$ Les lacunes, nul ne les perçoit ni ne les admet plus volontiers que l'auteur: de là son souhait qu'à la suite de recherches nouvelles, "le vieillissement viendra vite" pour son livre.

C'est cette optique qui confère son sens à notre travail. Il nous a paru essentiel de creuser, de compléter et de nuancer la perspective que Ouellet a aménagée à l'intérieur de son système pour la période 1792-1812: années cruciales de restructuration de l'économie canadienne, pour lesquelles il y a encore "pénurie relative" de données; période-clé par conséquent, en bonne partie inexplorée, qui réclame une prospection minutieuse. Or, à notre sens, l'on peut faire progresser le débat de deux manières. D'abord, il faut combler les brèches importantes dans les séries chronologiques de Ouellet sur cette période, au moyen de sources nouvelles ou supplémentaires qui semblent receler la clé de certaines énigmes. Il s'agit non seulement de compléter ces séries chronologiques, mais aussi d'en étendre l'éventail et d'en améliorer la qualité. Ensuite, même au prix de l'abandon temporaire d'une certaine "globalité", il faudrait dépasser "l'histoire sérielle" pour tendre à une véritable écono-

${ }^{5}$ Cf. Jean Hamelin et Fernand Ouellet, "Le Mouvement des prix agricoles dans la province de Québec: 1760-1851", in Claude Galarneau et Elzéar Lavoie, éd., La France et le Canada français $d u$ XVIe au $X X^{e}$ siècle (Québec, 1966), 35-39.

${ }^{6}$ Cf. Fernand Braudel, "Pour une histoire sérielle: Séville et l'Atlantique (1504-1650)", Annales E.S.C.., 18 (1963) : 541-553; P. Vilar, "Pour une meilleure compréhension entre économistes et historiens: 'Histoire quantitative' ou économétrie rétrospective", "Revue historique, no 223 (1965) : 293-312. 
mie historique: à l'énumération, la description et l'explication sommaire de chiffres et cle courbes, il faudrait substituer une véritable analyse économique, guidée bien sûr par une connaissance aussi approfondie que possible des divers plans de la société canadienne au cours de ces années.

On ne saurait concrétiser aisément un programme aussi ambitieux, encore moins en quelques mois. Aussi nous sommesnous contentés jusqu'ici de: soumettre certains résultats préliminaires au dernier Colloque de statistique de l'Association canadienne des Sciences politicues, en juin 1967.7 Nous ne sommes pas encore prêts à rédiger une étude systématique et détaillée sur l'économie historique de la période. Toutefois, nos travaux nous permettent déjà d'éliminer les "trous" dans plusieurs séries chronologiques présentées sous forme de graphiques dans l'ouvrage de Ouellet. $^{8}$ En outre, nous avons pu localiser des sources nouvelles qui rerdent désormais possible l'utilisation méthodique de prix officiels très diversifiés, sur le marché de Québec à tout le moins, sans l'inconvénient de collections incertaines à partir de sources plus ou moins sûres et déficientes. Comme ces prix mensuels englobent un ensemble très varié de produits, nous avons cru opportun d'attirer l'attention des chercheurs sur cette mine de renseignements chiffrés.

La première section de cet article comprendra des séries courtes sur les exportations et les importations du Bas-Canada, qui complètent les séries de Ouellet. Puis, nous extrairons quelques séries de prix officiels et illustrerons certaines utilisations possibles de cette source inédite. Enfin, un exposé succinct

${ }^{7}$ Gilles Paquet et Jean-Pierre Wallot, "Lower Canada, 1792-1812: Elements for a Quantitative Study (Preliminary Findings)", communication présentée au Colloque de statistique de l'ACSP, à Ottawa, le 10 juin 1967. On peut se procurer une copie de la communication en s'adressant aux auteurs.

8 En ce qui a trait aux inportations et aux exportations, notamment, les graphiques de Ouellet comportent des vides plus ou moins importants, entre 1792 et 1808 . Les rapports des officiers du port de Québec, en effet, semblent avoir disparu entre 1.792 et 1801 , puis manquent de régularité. A compter de 1809, cependant, le Journal de la Chambre d'Assemblée fournit tous les renseignements pertinents. Nous avons pu dénicher des récapitulations détaillées depuis 1793 jusqu'à 1808, pour les exportations, et de 1793 à 1802, pour les importations. 
définira le cadre de référence qui sous-tend nos travaux en cours sur la période 1792-1812.

\section{I - Le Commerce international du Bas-Canada à Québec}

$\mathrm{Au}$ tournant du XIX $\mathrm{X}^{e}$ siècle, le Bas-Canada s'engrène dans un ensemble assez complexe d'échanges internationaux. Une bonne compréhension des mécanismes de restructuration de l'économie du Bas-Canada, entre 1790 et 1810, nécessite donc une connaissance aussi précise que possible de ce réseau. D'où l'importance capitale des données qui manquaient à Ouellet pour une analyse à la fois souple et "globale" des changements structuraux qui s'accomplissent dans la première décennie du siècle dernier. Pour le moment, nous nous contentons d'exposer et de commenter brièvement un indicateur global d'activité économique internationale: le nombre et le tonnage des navires qui arrivent au port de Québec ou qui le quittent (tableau I). Certes, nous n'épuisons pas le problème! Cet indicateur, il faudrait en triturer tous les éléments à l'aide d'un ordinateur, et il ne tient compte que des entrées et sorties à Québec. Outre le contexte impérial et mondial, l'on ne saurait négliger les échanges, même s'ils sont beaucoup moins importants, entre le Haut et le Bas-Canada, entre ces deux colonies et les États-Unis, notamment à Saint-Jean. Mais même une mesure globale imparfaite suggère un ordre de grandeur et une structure chronologique pour cette période.

Ainsi, on peut découper les années 1793 à 1810 en trois sous-périodes. De 1793 jusqu'à 1800, le tonnage des navires quittant Québec se situe aux environs de 17,000 tonneaux. Dès 1801 et jusqu'en 1806, il se hausse à un palier différent, soit environ 30,000 tonneaux. Ces deux sous-périodes s'articulent sur le commerce des fourrures, les produits agricoles et un démarrage modeste du bois à compter de 1801-1802. Il est facile de remonter de ces pulsations de la conjoncture aux forces sous-jacentes, soit les commerces mentionnés. ${ }^{9}$ De 1807 à 1810,

9 Cf. Fernand Ouellet, op. cit., ch. 6; Hugh Gray, Letters from Canada... (London, 1809), "Letter XV". 
TABLEAU I 1

Entrées et sorties à Québec
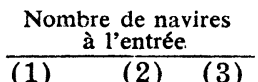

Tonnage à l'entrée

(1) (2) (3)

Nombre de navires

$$
\frac{\text { à la sortie }}{\text { (1) (2) (3) }}
$$

Tonnage à la sortie

$\frac{\text { Tonnage à la sortie }}{(1) \quad(2)}$

\begin{tabular}{|c|c|c|c|}
\hline 1793 : & 113 & - & - \\
\hline 1794: & 114 & - & - \\
\hline 1795: & 117 & - & - \\
\hline 1796: & 67 & - & - \\
\hline 1797: & 88 & - & - \\
\hline 1798: & 78 & - & - \\
\hline 1799 : & 125 & 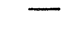 & - \\
\hline 1800 : & 140 & - & - \\
\hline 1801: & $\begin{array}{l}175 \\
198\end{array}$ & $\overline{196}$ & $\overline{196}$ \\
\hline 1803 : & - & - & \\
\hline 1804: & - & 162 & 162 \\
\hline 1805 : & - & $134^{*}$ & $135^{*}$ \\
\hline 1806 : & - & 186 & 186 \\
\hline 1807: & - & 212 & 213 \\
\hline $1808:$ & & 295 & 292 \\
\hline 1809 & 434@ & 426 & 424 \\
\hline 1810: & 635@ & 629 & 631 \\
\hline 1811: & 495@ & 496 & - \\
\hline 1816 & 362@ & 351 & - \\
\hline
\end{tabular}

$\begin{array}{ccc}15,271 & - & - \\ 16,827 & - & - \\ 18,023 & - & - \\ 11,050 & - & - \\ 13,349 & - & - \\ 11,882 & - & - \\ 17,941 & - & - \\ 16,837 & \overline{-} & - \\ 25,736 & 33,232 & 32,728^{1 / 2} \\ 33,139 & \overline{-} & 25,015 \\ - & 25,021 & 20,847^{*} \\ - & 21,333^{*} & 31,920 \\ - & 33,104 & 38,448 \\ - & 37,9621 / 2 & 38,781 \\ - & 60,783 & 60,781 \\ 87,825 @ & 68,383^{* *} & 86,139 \\ 138,057 @ & 132,9601 / 2 & 132,696 \\ 107,075 @ & 106,5301 / 2 & - \\ 77,100 @ & 76,306 & -\end{array}$

118 - -

\section{8,715}

16,126

$116-128-19,126$
$71-1,745$

$71-11,789$
$105 \quad-\quad 15,668$

$91-13,939$

$138 \quad-\quad-\quad 19,537$

153

-

$\begin{array}{llll}188 & - & & 27,986 \\ 211 & 211 & 211 & 35,754\end{array}$

35,754

30,403

26,883

$173 \quad \overline{173} \quad \overline{169}$

$170129 * 129 *$

$193 \quad 192 \quad 194$

26,506

33,996

-

-

334@327 334

(3)

661@654 644

532@ $526-$

-

143,793@

116,687@119,0181/2

$35, \overline{80} 61 / 2$

$26, \overline{595}$

26,730

$17,8161 / 2^{*} \quad 18,449$ *

34,187

34,130

$43,807 \frac{1}{2} \quad 44,207$

$71,179 \quad 71,233$

$87,964 \quad 88,056$

$77,100 @ 76,306$ -

1 Sources. (1): nous avons compilé les entrées (1793-1802) et sorties (1793-1808) à partir de trois récapitulations différentes qui se recoupent à une variante près: APC, M.G.11, Q.89: 247 (entrées et sorties, 1793-1802); ibid., Q.90: 3a (entrées et sorties, 1793-1802, avec des chiffres plus complets pour 1802); ibid., Q.109: 248a (sorties seulement, 1793-1808). Les Journaux de la Chambre d'Assemble du Basde la récapitulation en $\mathbf{Q} .109$ et ceux du Journal coincident pour 1808, nous avons inscrit les seconds dans les colonnes (1) de manière a constituer en quelque sorte une série "officielle".

(2): calcul du nombre de navires et du tonnage à partir des totaux au bas des rapports trimestriels de l'officier naval du port de Québec. Ces rapports sont envoyés régulièrement à Londres par l'administrateur de la colonie (série Q), bien qu'à compter de 1808 ils se trouvent rassemblés (registres de l'officier naval de Québec, Q.1296 ss).

(3): calcul du nombre de navires et du tonnage en prenant les navires un à un - nous nous sommes arrêtés pour le moment à 1810 à partir de la même source qu'en (2). On notera le faible écart tant entre les séries relatives au nombre de navires, souvent identiques, qu'entre celles de tonnage. Il sera donc possible de bâtir une série homogène.

$\dagger$ Variante improbable dans Q.89: 17,986.

* Chiffres incomplets: le dernier trimestre manque.

** L'officier du port a omis une page complète dans son rapport.

(a) Chiffres extraits des Journaux de la Chambre d'Assemblée du Bas-Canada. 
c'est l'explosion du commerce extérieur: les tonnages doublent de 1806 à 1808, puis de nouveau de 1808 à 1810. Ils fléchissent légèrement en 1811, en partie sous le coup d'une crise qui s'abat sur l'Angleterre au milieu de 1810, pour s'effondrer en 1812, année de guerre. ${ }^{10}$

Cette expansion du trafic maritime révèle une restructuration de l'économie qui n'est pourtant pas évidente à ce niveau des valeurs globales. L'importance absolue et relative des exportations de bois a déclenché cette ascension vertigineuse, et même au niveau des chiffres globaux, l'on peut déceler des indices de ce réajustement. D'une part, Harold Innis a noté la relation entre la mise en branle du commerce du bois et certains phénomènes de capacité excédentaire et de déséquilibre des cargaisons à l'entrée et à la sortie du port de Québec. ${ }^{11}$ D'autre part, les données que nous avons colligées sur les tonnages des navires révèlent en outre qu'on peut jumeler la hausse de l'importance relative du lest dans les navires remontant le SaintLaurent à l'accroissement marqué de la dimension moyenne des navires. Le tableau II illustre bien cette relation qui s'avère statistiquement significative. ${ }^{12}$ On peut donc voir que la restructuration des échanges est liée à certains changements dans la technologie des transports maritimes qu'il faudrait étudier plus longuement.

Toutefois, il faut ventiler quelque peu les séries de tonnages afin de bien discerner et d'évaluer les ajustements. Cette

10 Nous n'avons pas voulu incorporer les années 1812-1815, années de guerre qui bouleversent l'agriculture et l'économie du Bas-Canada, dans un cycle "1804-1815" de manière à nous conformer à l'historiographie européenne. Hamelin et Ouellet ont cru bon de le faire (cf. "Le Mouvement des prix agricoles", in C. Galarneau et E. Lavoie, éd., op. cit., 42).

11 H.A. Innis, "Unused Capacity as a Factor in Canadian Economic History", Canadian Journal of Economics and Political Science, 2 (1936): 1-15.

12 Nous avons calculé le coefficient de corrélation de rang de Spearman entre le lest comme pourcentage du tonnage à l'entrée du port de Québec et la dimension moyenne des navires quittant Québec par trimestre de 1802 à 1810 . Le coefficient est de l'ordre de .7 et s'avère significatif au niveau de $1 \%$. En d'autres termes, il y a moins d'une chance sur cent que l'on obtienne un résultat comme celui que nous avons observé si en fait ces deux variables sont indépendantes. 
T'ABLEAU II

Lest en pourcentage du tonnage et tonnage moyen des navires

\begin{tabular}{|c|c|c|c|}
\hline & & $\begin{array}{l}\text { Lest en pourcentage } \\
\text { du tonnage à l'entrée } \\
\text { du port de Québec }\end{array}$ & $\begin{array}{r}\text { Tonnage } \\
\text { des navires } \\
\text { du port de }\end{array}$ \\
\hline $1802:$ & $\begin{array}{l}\text { Avril-juillet } \\
\text { Juillet-octobre } \\
\text { Octobre-janvier }\end{array}$ & $\begin{array}{l}.137 \\
.286 \\
.396\end{array}$ & $\begin{array}{r}156 \\
96 \\
185\end{array}$ \\
\hline $1803:$ & Les données man & quent. & \\
\hline $1804:$ & $\begin{array}{l}\text { Avril-juillet } \\
\text { Juillet-octobre } \\
\text { Octobre-janvier }\end{array}$ & $\begin{array}{l}.236 \\
.231 \\
.289\end{array}$ & $\begin{array}{r}98 \\
153 \\
189\end{array}$ \\
\hline $1805:$ & $\begin{array}{l}\text { Avril-juillet } \\
\text { Juillet-octobre } \\
\text { Octobre-janvier }\end{array}$ & $\begin{array}{l}.307 \\
.336 \\
-\end{array}$ & $\begin{array}{l}116 \\
177\end{array}$ \\
\hline $1806:$ & $\begin{array}{l}\text { Avril-juillet } \\
\text { Juillet-octobre } \\
\text { Octobre-janvier }\end{array}$ & $\begin{array}{l}.291 \\
.712 \\
.381\end{array}$ & $\begin{array}{l}131 \\
189 \\
202\end{array}$ \\
\hline $1807:$ & $\begin{array}{l}\text { Avril-juillet } \\
\text { Juillet-octobre } \\
\text { Octobre-janvier }\end{array}$ & $\begin{array}{l}.449 \\
.457 \\
.479\end{array}$ & $\begin{array}{l}150 \\
195 \\
225\end{array}$ \\
\hline $1808:$ & $\begin{array}{l}\text { Avril-juillet } \\
\text { Juillet-octobre } \\
\text { Octobre-janvier }\end{array}$ & $\begin{array}{l}.639 \\
.648 \\
-\end{array}$ & $\begin{array}{l}153 \\
182 \\
-\end{array}$ \\
\hline $1809:$ & $\begin{array}{l}\text { Avril-juillet } \\
\text { Juillet-octobre } \\
\text { Octobre-janvier }\end{array}$ & $\begin{array}{l}.536 \\
.699 \\
.588\end{array}$ & $\begin{array}{l}21 \\
20\end{array}$ \\
\hline $1810:$ & $\begin{array}{l}\text { Avril-juillet } \\
\text { Juillet-octobre } \\
\text { Octobre-janvier }\end{array}$ & $\begin{array}{l}.819 \\
.748 \\
.712\end{array}$ & $\begin{array}{l}264 \\
201\end{array}$ \\
\hline
\end{tabular}

Source: A.P.C., registres de l'officier naval (M.G. 11, série Q). ventilation peut s'opérer dans plusieurs directions: découpage trimestriel, décomposition sectorielle des exportations et des importations, analyse des flux selon les lieux d'origine et de destination. Nous nous contentons de livrer ici l'évolution des quantités exportées et importées de certains biens et produits impor ants - chiffres qui justement comblent des lacunes dans les séries de Ouellet (voir tableaux III et IV). Nous réservons pour une communication ultérieure une ventilation plus précise des flux qui tissent le réseau des relations internationales $\mathrm{du}$ Bas-Canada au tournant du $\mathrm{XIX}^{\mathrm{e}}$ siècle.

Nous ne saurions tenter de décrire ici en détail la restructuration de la composition sectorielle du commerce international du Bas-Canada, de 1790 à 1810 environ. Cependant, 


\section{TABLEAU III}

Exportations depuis le port de Québec (1793-1808)*

\begin{tabular}{|c|c|c|c|c|c|c|}
\hline & $\frac{\text { Blé }}{(\text { minots) }}$ & $\frac{\text { Farine }}{\text { (barils) }}$ & $\frac{\text { Biscuits }}{\text { (cwts) }}$ & $\frac{\text { Avoine }}{\text { (minots) }}$ & $\frac{\text { Orge }}{\text { (minots) }}$ & $\frac{\text { Poids }}{\text { (minots) }}$ \\
\hline $\begin{array}{l}\text { 1793: } \\
1794: \\
1795: \\
1796: \\
1797: \\
1798: \\
1799: \\
1800: \\
1801: \\
1802: \\
1803: \\
1804: \\
1805: \\
1806: \\
1807: \\
1808:\end{array}$ & $\begin{array}{r}487,099 \\
414,551 \\
394,752 \\
3,106 \\
31,449 \\
91,727 \\
128,870 \\
217,125 \\
472,723 \\
1,010,033 \\
360,892 \\
201,543 \\
22,016 \\
96,908 \\
231,543 \\
186,708\end{array}$ & $\begin{array}{r}10,916 \\
13,787 \\
17,967 \\
4,352 \\
13,932 \\
9,530 \\
14,475 \\
20,271 \\
38,146 \\
28,301 \\
15,432 \\
14,319 \\
18,590 \\
10,997 \\
20,442 \\
42,462\end{array}$ & $\begin{array}{r}9,822 \\
15,047 \\
19,722 \\
3,882 \\
8,147 \\
12,165 \\
21,535 \\
24,623 \\
32,303 \\
22,051 \\
17,581 \\
21,255 \\
26,462 \\
23,659 \\
28,047 \\
32,587\end{array}$ & $\begin{array}{l}3,043 \\
1,135 \\
1,129 \\
1, \overline{490} \\
1,715^{* *} \\
476 \\
5,517 \\
5,013 \\
1,898 \\
1,462 \\
2,981 \\
5,016 \\
2,734 \\
2,369 \\
2,669\end{array}$ & $\begin{array}{l}= \\
= \\
= \\
6 \\
70 \\
1,382 \\
3,744 \\
5,585 \\
457 \\
4,253 \\
6,911 \\
5,158 \\
5,010 \\
5,994\end{array}$ & $\begin{array}{r}180 \\
832 \\
3,189 \\
271 \\
1,226 \\
3,244 \\
495 \\
3,193 \\
6,559 \\
424 \\
1,605 \\
286 \\
4,155 \\
6,191 \\
7,181 \\
52,934\end{array}$ \\
\hline
\end{tabular}

\begin{tabular}{|c|c|c|c|c|c|c|}
\hline $\begin{array}{c}\text { Pommes de } \\
\text { terre }\end{array}$ & $\begin{array}{c}\text { Graine } \\
\text { de lin }\end{array}$ & Sel & Bœuf & Bœuf & Porc & Porc \\
\hline (minots) & (minots) & (minots) & (tierşons) & (barils) & (tierçons) & (barils) \\
\hline- & 17,465 & - & - & 345 & - & - \\
\hline & $\begin{array}{r}9,225 \\
11,460\end{array}$ & 6,800 & $\begin{array}{r}20 \\
-\quad\end{array}$ & 607 & - & - \\
\hline $\begin{array}{r}1,570 \\
980\end{array}$ & $\begin{array}{l}11,460 \\
10,302\end{array}$ & $=$ & $=$ & $\begin{array}{r}1,451 \\
927\end{array}$ & - & - \\
\hline $\begin{array}{r}980 \\
1,248\end{array}$ & 5,058 & $\overline{4,541}$ & 二 & 370 & $=$ & 二 \\
\hline 1,098 & 5,317 & - & - & 590 & - & - \\
\hline 1,250 & 2,339 & 220 & - & 1,248 & - & - \\
\hline 1,375 & 326 & - & 一 & 516 & - & - \\
\hline- & 9,079 & 9,916 & - & 1,412 & - & - \\
\hline 一 & 5,102 & 7,008 & 66 & $1,199^{* *}$ & - & - \\
\hline$\rightarrow$ & 8,224 & - & 1,047 & 1,908 & 6 & 1,924 \\
\hline 48 & 10,778 & 8,808 & 593 & 908 & 17 & 1,926 \\
\hline 140 & 6,523 & - & 10 & 1,564 & 74 & 696 \\
\hline & 3,577 & - & - & 808 & 26 & 1,077 \\
\hline - & 8,680 & 1,500 & 9 & & 108 & 1,043 \\
\hline - & 13,530 & 300 & - & 1,509 & 179 & 1,732 \\
\hline
\end{tabular}

* Sources: récapitulations 1793-1802 (APC, M.G.11, Q.89: 247: Q.90: $3 a)$; $1793-1808(Q .109: 248 a)$. Nous avons adopté les chiffres de la compilation plus récente (Q.109).

** Variantes: 1793: 23,225 (Q.89) et 23,223 (Q.90) visons. 1794: 4 mâts (Q.89 et 90$)$. 1797: 15 animaux (Q.89 et 90$)$. 1798: 2,715 minots d'avoine (Q.89); 154 animaux (Q. 89 et 90). 1799: 8 animaux (Q.89 et 90). 1801: 205 animaux (Q. 89 et 90). 1802: 494 pi., 2,000 madriers de chêne (Q.90); 1,265 barils de bœuf (Q.90); 627,906 (Q.89) et 597,062 (Q.90) douves; 184 animaux (Q. 89 et 90$)$. 


\begin{tabular}{|c|c|c|c|c|c|c|}
\hline & $\frac{\begin{array}{c}\text { Beurre et } \\
\text { lard }\end{array}}{\text { et caquins }}$ & $\begin{array}{c}\begin{array}{c}\text { Poisson } \\
\text { séché }\end{array} \\
\text { (cwts) }\end{array}$ & $\frac{\text { Chêne }}{\text { (pièces) }}$ & $\frac{\text { Pin }}{(\text { pièces) }}$ & $\begin{array}{l}\text { Madriers } \\
\text { de chêne }\end{array}$ & $\begin{array}{c}\text { Bouts de } \\
\text { douves } \\
\text { en chêne } \\
\frac{\text { (pièces) }}{}\end{array}$ \\
\hline 1793: & - & 2,918 & 1,240 & - & - & - \\
\hline 1794: & 14 & 1,601 & 729 & - & - & - \\
\hline 1795: & 553 & 1,517 & 316 & 620 & 500 & - \\
\hline 1796: & 20 & 330 & 1,394 & 11 & 372 & - \\
\hline 1797: & 50 & 52 & 2,888 & 255 & 45 & - \\
\hline 1798: & 905 & 1,720 & 2,221 & 3 & 19 & - \\
\hline 1799: & 250 & 5,716 & 2,299 & 104 & 105 & 1,400 \\
\hline $1800:$ & 389 & 702 & 1,633 & 12 & 1,087 & - \\
\hline 1801: & 531 & 854 & 1,268 & 1,061 & 494 & - \\
\hline 1802: & 426 & 415 & 1,607 & 1,035 & $200 * *$ & 10,113 \\
\hline 1803: & 95 & 460 & 3,819 & 3,153 & 680 & 8,030 \\
\hline 1804: & 242 & 2,825 & 4,229 & 873 & 135 & 836 \\
\hline 1805: & 199 & 3,118 & 4,535 & 896 & 369 & 17,842 \\
\hline 1806: & 186 & 1,904 & 8,178 & 2,130 & 885 & 13,688 \\
\hline 1807: & 418 et 502 & 976 & 11,195 & $\mathbf{3}, 333$ & 1,274 & 47,810 \\
\hline 1808: & 3,043 & 2,949 & 12,372 & 14,510 & 209 & 62,453 \\
\hline
\end{tabular}

\begin{tabular}{|c|c|c|c|c|c|c|}
\hline & Bardeaux & $\begin{array}{c}\text { Madriers } \\
\text { et } \\
\text { planches } \\
\text { de pin } \\
\text { (pièces) }\end{array}$ & $\begin{array}{c}\begin{array}{c}\text { Douves et } \\
\text { hauts bouts } \\
\text { de chêne }\end{array} \\
\text { (pièces) }\end{array}$ & $\begin{array}{l}\text { Lattes de } \\
\text { noyer }\end{array}$ & Mâts & $\frac{\text { Cerceaux }}{2^{\prime} \text { de long) }}$ \\
\hline 1793: & 139,970 & 22,059 & 294,608 & - & 14 & $\begin{array}{l}33,775 \\
74,600\end{array}$ \\
\hline 1794: & 90,930 & 15,863 & 151,354 & - & $3^{* *}$ & $\begin{array}{l}74,600 \\
27,000\end{array}$ \\
\hline $\begin{array}{l}\text { 1795: } \\
1796:\end{array}$ & E & $\begin{array}{l}30,00 \\
18,28\end{array}$ & $\begin{array}{l}593,652 \\
818,799\end{array}$ & 二 & & $\begin{array}{l}27,000 \\
18,550\end{array}$ \\
\hline 1797: & 三 & $\begin{array}{l}18,280 \\
47691\end{array}$ & 734,198 & E & 3 & $\begin{array}{r}18,000 \\
7,100\end{array}$ \\
\hline 1798: & - & 11,769 & 446,469 & - & 4 & 37,600 \\
\hline 1799: & - & 52,140 & 703,778 & 150 & - & 76,90 \\
\hline 800 & - & 34,863 & 542,656 & - & 4 & 27,00 \\
\hline 801. & 67,000 & 60,033 & 535,547 & - & 57 & 11,50 \\
\hline 802: & 78,900 & 104,735 & $586,949 * *$ & - & 34 & 57,2 \\
\hline 1803 & 34,150 & 124,197 & 763,668 & - & 283 & $92,8^{\prime}$ \\
\hline & 4,000 & 78,391 & $1,123,958$ & - & 115 & 109,8 \\
\hline 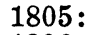 & 3,000 & 42,820 & $1,043,994$ & - & 103 & 119,6 \\
\hline & 16,000 & 66,116 & $1,808,234$ & 1,778 & 332 & 140,00 \\
\hline & 29,000 & 106,363 & $1,783,890$ & 11,579 & 973 & 88,2 \\
\hline & 60,500 & 194,467 & $1,824,861$ & 130,915 & 3,994 & 215,5 \\
\hline
\end{tabular}

pareils renseignements sont nécessaires à la reconstitution de la balance commerciale de la colonie durant cette période. Pour ce faire, il nous faudra amasser une quantité beaucoup plus considérable de prix pour les principales denrées, notamment avant 1805. Nous sommes loin d'avoir accumulé les données requises pour de tels calculs. 


\begin{tabular}{|c|c|c|c|c|c|c|}
\hline & $\frac{\text { Rames }}{\text { (paires) }}$ & $\frac{\text { Chanvre }}{\text { (ballots) }}$ & $\frac{\text { Animaux }}{\text { (têtes) }}$ & $\begin{array}{l}\text { Potasse et } \\
\text { Perlasse } \\
\text { (cwt) }\end{array}$ & Castors & Martins \\
\hline $\begin{array}{l}1793: \\
1794: \\
1795: \\
1796: \\
1797: \\
1798: \\
1799: \\
1800: \\
1801: \\
1802: \\
1803: \\
1804: \\
1805: \\
1806: \\
1807: \\
1808:\end{array}$ & $\begin{array}{l}\overline{3} \\
\overline{ } \\
\overline{ } \\
\overline{ } \\
\overline{1} \\
1,159 \\
1,469 \\
401 \\
611 \\
836 \\
188 \\
207 \\
88 \\
3,361\end{array}$ & $\begin{array}{l}= \\
= \\
= \\
= \\
= \\
= \\
= \\
\\
19\end{array}$ & $\begin{array}{c}58 \\
173 \\
2 \\
10 \\
125^{* *} \\
44^{* *} \\
37^{* *} \\
32 \\
225^{* *} \\
190^{* *} \\
144 \\
111 \\
142 \\
169 \\
23 \\
303\end{array}$ & $\begin{array}{r}9,702 \\
7,824 \\
7,746 \\
12,358 \\
14,933 \\
22,051 \\
24,355 \\
29,142 \\
25,620 \\
16,920 \\
17,896 \\
21,946 \\
27,291 \\
36,450 \\
46,948 \\
107,652\end{array}$ & $\begin{array}{r}182,346 \\
155,599 \\
144,945 \\
130,820 \\
124,612 \\
127,440 \\
117,165 \\
135,043 \\
119,965 \\
144,189 \\
93,778 \\
111,448 \\
92,003 \\
119,708 \\
114,363 \\
126,927\end{array}$ & $\begin{array}{l}26,680 \dagger \\
37,785 \\
45,214 \\
31,143 \\
54,218 \\
52,641 \\
35,267 \\
40,345 \\
24,451 \\
23,808 \\
31,441 \\
23,796 \\
14,275 \\
55,687 \\
46,801 \\
9,530\end{array}$ \\
\hline
\end{tabular}

\begin{tabular}{|c|c|c|c|c|c|c|}
\hline & Chevreuils & $\begin{array}{l}\text { Ratons } \\
\text { laveurs }\end{array}$ & Loutres & Visons & $\begin{array}{c}\text { Rats } \\
\text { musqués }\end{array}$ & Poêles \\
\hline 1793: & 126,505 & 190,476 & 22,758 & $23,233^{* *}$ & 32,659 & 1 \\
\hline 1794: & 130,513 & 125,781 & 17,387 & 12,837 & 28,230 & - \\
\hline $\begin{array}{l}\text { 1795: } \\
\text { 1796: }\end{array}$ & $\begin{array}{l}165,483 \\
168,035\end{array}$ & $\begin{array}{r}124,839 \\
135,835\end{array}$ & $\begin{array}{l}18,533 \\
16,107\end{array}$ & $\begin{array}{l}8,946 \\
9,693\end{array}$ & $\begin{array}{l}83,009 \\
96,263\end{array}$ & $=$ \\
\hline $\begin{array}{l}1796: \\
1797:\end{array}$ & 171,424 & 189,992 & 16,600 & $\begin{array}{r}5,000 \\
10,690\end{array}$ & 181,520 & = \\
\hline 1798: & 161,486 & 194,237 & 15,848 & 9,128 & 71,506 & - \\
\hline 7 & 173,065 & 135,468 & 16,966 & 8,326 & 8,776 & 44 \\
\hline 3 & 204,587 & 110,984 & 19,109 & 8,428 & 12,397 & - \\
\hline 801 & 227,205 & 92,345 & 21,837 & 10,689 & - & - \\
\hline 802: & 184,388 & 147,882 & 19,855 & 11,148 & 26,289 & 74 \\
\hline : & 206,979 & 152,333 & 17,465 & 12,062 & 78,625 & $\begin{array}{l}56 \\
40\end{array}$ \\
\hline$\vdots$ & $\begin{array}{l}249,050 \\
213,843\end{array}$ & $\begin{array}{l}178,479 \\
124,319\end{array}$ & $\begin{array}{l}20,237 \\
15,246\end{array}$ & $\begin{array}{l}11,567 \\
11,431\end{array}$ & $\begin{array}{l}94,199 \\
66,127\end{array}$ & $\begin{array}{r}40 \\
224\end{array}$ \\
\hline & 212,037 & 125,622 & 12,297 & 14,872 & 16,849 & - \\
\hline & 166,366 & 79,174 & 8,390 & 9,214 & 1,750 & 161 \\
\hline & 103,875 & 123,307 & 7,230 & 9,108 & & 127 \\
\hline
\end{tabular}

*** Bétail à cornes, chevaux, porcs, moutons.

† La dernière récapitulation donne 37,785 . Il s'agit sans doute d'une confusion avec le chiffre suivant. Les deux autres compilations concordent sur 26,680 .

\section{II - Les Prix officiels à Québec: une source riche et inexploitée}

Les travaux les plus importants sur les mouvements de prix au Canada, avant 1850, ont été accomplis par J. Hamelin et $\mathrm{F}$. Ouellet. Utilisant les rapports sporadiques des journaux et les livres de comptes de communautés religieuses, ils ont déployé une ingéniosité remarquable dans la préparation de 
T.ABLEAU IV

Importations au port de Québec (1793-1802)*

\begin{tabular}{|c|c|c|c|c|}
\hline & $\frac{\text { Vin de Madère }}{\text { (galls) }}$ & $\frac{\text { Autres vins }}{\text { (galls) }}$ & $\begin{array}{l}\begin{array}{c}\text { Brandy et Gin } \\
\text { étrangers }\end{array} \\
\text { (galls) }\end{array}$ & $\frac{\text { Rhum }}{\text { (galls) }}$ \\
\hline $\begin{array}{l}1793: \\
1794: \\
1795: \\
1796: \\
1797: \\
1798: \\
1799: \\
1800 \vdots \\
1801: \\
1802:\end{array}$ & $\begin{array}{l}42,680 \\
12,705 \\
18,861 \\
20,804 \\
25,561 \\
17,031 \\
17,370 \\
22,723 \\
52,949 \\
21,474\end{array}$ & $\begin{array}{r}151,320 \\
75,655 \\
102,729 \\
132,699 \\
65,585 \\
208,474 \\
136,012 \\
137,214 \\
108,314 \\
170,980\end{array}$ & $\begin{array}{r}660 \\
3,730 \\
326 \\
1,298 \\
16,043 \\
11,402 \\
32,957 \\
37,351 \\
7,144 \\
13,806\end{array}$ & $\begin{array}{l}320,620 \\
391,370 \\
116,124 \\
248,569 \\
162,684 \\
240,584 \\
492,969 \\
195,777 \\
302,930 \\
384,937\end{array}$ \\
\hline
\end{tabular}

\begin{tabular}{|c|c|c|c|c|}
\hline & $\frac{\text { Mélasse }}{\text { (galls) }}$ & $\frac{\text { Pain de sucre }}{\text { (livres) }}$ & $\begin{array}{c}\text { Sucre } \\
\text { "Muscovado" } \\
\text { (livres) }\end{array}$ & $\frac{\text { Café }}{\text { (livres) }}$ \\
\hline $\begin{array}{l}1793: \\
1794: \\
1795: \\
1796: \\
1797: \\
1798: \\
1799: \\
1800: \\
1801: \\
1802:\end{array}$ & $\begin{array}{l}25,740 \\
50,220 \\
17,767 \\
62,898 \\
63,525 \\
19,422 \\
69,165 \\
37,073 \\
65,693 \\
49,094\end{array}$ & $\begin{array}{c}18,650 \\
17,874 \\
107,1801 / 2 \\
120,656 \\
94,080 \\
141,990 \\
126,399 \\
91,831 \\
67,938 \\
165,988\end{array}$ & $\begin{array}{r}107,520 \\
125,431 \\
76,463 \\
126,132 \\
44,458 \\
158,414 \\
143,384 \\
233,152 \\
499,387 \\
426,891\end{array}$ & $\begin{array}{l}16,540 \\
11,450 \\
18,404 \\
75,746 \\
21,814 \\
34,080 \\
48,847 \\
35,079 \\
50,816 \\
29,359\end{array}$ \\
\hline
\end{tabular}

\begin{tabular}{|c|c|c|c|c|}
\hline & $\begin{array}{l}\text { Tabac en } \\
\text { feuille }\end{array}$ & $\begin{array}{c}\begin{array}{c}\text { Tabac } \\
\text { manufacturé }\end{array} \\
\text { (livres) }\end{array}$ & $\frac{\text { Cartes à jouer }}{\text { (paquets) }}$ & (minots) \\
\hline $\begin{array}{l}1793: \\
1794: \\
1795: \\
1796: \\
1797: \\
1798: \\
1799: \\
1800: \\
1801: \\
1802:\end{array}$ & $\begin{array}{r}82,600 \\
7,987 \\
244,975 \\
149,547 \\
84,747 \\
163,961 \\
79,500 \\
158,258 \\
84,148 \\
140,692\end{array}$ & $\begin{array}{l}\bar{Z} \\
\bar{z} \\
\bar{z} \\
\overline{4} \\
46,283\end{array}$ & \begin{tabular}{r}
\multicolumn{1}{c}{} \\
55,360 \\
3,696 \\
17,419 \\
22,308 \\
13,736 \\
10,116 \\
7,020 \\
17,400
\end{tabular} & $\begin{array}{c}150,732 \\
58,004 \\
35,450 \\
31,6061 / 2 \\
38,513 \\
69,444 \\
83,536 \\
102,975 \\
136,010 \\
101,0641 / 2\end{array}$ \\
\hline
\end{tabular}

* Source: APC, M.G.11, Q.89: 247; Q.90: 3a. Valeur annuelle moyenne: de $£ 300,000$ à $£ 330,000$ sterling. 
séries chronologiques de prix agricoles. ${ }^{13}$ Toutefois, ces données sont loin d'être satisfaisantes pour les besoins d'une analyse affinée. En effet, "durant les années antérieures à 1812 pour Québec et 1828 pour Montréal... il nous a fallu nous contenter souvent de la moyenne de quatre ou cinq chiffres répartis sur les quatre saisons", écrivent-ils. ${ }^{14} \mathrm{Si}$ utiles qu'elles soient, ces reconstructions fragiles revêtent l'aspect séduisant d'une trompeuse certitude. Les deux chercheurs ont d'ailleurs indiqué clairement des limites d'un tel exercice et procédé avec prudence dans la discussion de leurs séries. Cependant, malgré toutes les mises en garde, une courbe même "simplette" ${ }^{15}$ s'étale avec un air sérieux de fausse précision qui obnubile trop facilement l'esprit critique d'historiens souvent peu rompus aux techniques statistiques. Il faut donc chercher à améliorer, si possible, la qualité de ces séries, à élargir leur éventail et à étendre leur champ d'application.

Or, ainsi que Hamelin et Ouellet l'ont dit,"à moins qu'un chercheur ne mette la main sur quelques sources inédites, les prix agricoles demeureront les seuls indices sériés d'une histoire globale des prix durant cette période (1760-1851)" ${ }^{16}$. Ce pas est maintenant franchi en partie. Il existe en effet des séries de prix préparées par le clerc du marché à Québec et expédiées mensuellement à Londres à compter de 1805 . Il devient donc possible dès maintenant de suivre la courbe des prix à Québec, chaque mois, pour plus de 70 produits agricoles et non-agricoles. ${ }^{17}$ On conçoit aisément la valeur capitale de cette source nouvelle et ses avantages sur les anciennes. Premièrement, les

$13 \mathrm{~J}$. Hamelin et F. Ouellet, "Le mouvement des prix agricoles...", in C. Galarneau et E. Lavoie, éd., op.cit., 35-48.

14 Ibid., 38.

15 Nous ne pouvons souscrire à la position excessive de Robert Mandrou pour qui "la construction d'une courbe - fût-elle simplette - semble préférable à une fine page de définitions". ("Mathématiques et histoire", Critica storica, 1 (1962): 39 ).

$16 \mathrm{~J}$. Hamelin et F. Ouellet, "Le mouvement des prix agricoles...", in C. Galarneau et E. Lavoie, éd., op.cit., 36.

17 Ces prix se trouvent dans la correspondance officielle de l'administrateur de la colonie: cf. APC, M.G. 11, série Q, v.g. Q. 97: 143 ss; Q. 98: 183 ss, 220 ss; etc. Dans ce cas, comme dans celui des récapitulations (tableaux III et IV), l'on peut s'étonner qu'une source aussi fondamentale et accessible ait été si délaissée en histoire économique. 
prix touchent à peu près tout ce qui se vend sur le marché, depuis le blé jusqu'au charbon, le rhum et les chandelles. Deuxièmement, il s'agit de prix officiels sur le marché, donc de prix de détail. La plupart des prix rassemblés jusqu'ici consistaient surtout en "prix aux livres", où l'on départageait difficilement prix de gros et prix de détail. En outre, Ouellet et Hamelin ont extrait leurs prix de comptabilités privées et de livres de comptes d'institutions religieuses. Dans le cas de ces dernières, en particulier, il est difficile de déterminer l'écart entre ces prix et ceux payés par les consommateurs ordinaires sur les marchés. On a cependant noté un biais systématique. ${ }^{18}$ Ces prix aux livres pouvaient résulter de l'influence d'une double série de forces: d'une part, les mécanismes normaux du marché, et d'autre part, les interventions d'agents économiques qui n'avaient rien d'“atomistique". Par exemple, l'on peut imaginer deux politiques "charitables": l'une qui aurait haussé les prix en période de récession et freiné leur hausse en période d'expansion, de manière c̀ redistribuer les revenus des censitaires dans le temps; une autre, celle relevée par Hamelin et Ouellet, en sens inverse, i.e. qui aurait combattu la hausse des prix ou la cherté des vivres en temps de crise. Or, les prix au marché échappent à cette "composante religieuse" et aux incertitudes de la comptabilité "au livre". ${ }^{19}$ Troisièmement, ces prix officiels, disponibles sur une base mensuelle, permettent l'établissement d'indices précis et une étude de la variation saisonnière des prix dans le Basi-Canada. Enfin, d'autres chercheurs pourront puiser à la même source pour les années postérieures à 1812: ceci permettrait une étude documentée des prix à la consommation et du niveau de vie sur une longue période.

Il semble donc que l'on puisse affiner considérablement notre compréhension des phénomènes économiques, dans la

18 Hamelin et Ouellet mentionnent que les prix du Séminaire de Québec sont plus bas que ceux du marché et "moins sensibles que ceux du marché à la conjoncture locale et internationale" ("Le mouvement des prix agricoles", in C. Galarneau et E. Lavoie, éd., op.cit., 37). Mais ils n'analysent pas ce que représente l'écart entre les prix du marché et les prix aux livres.

19 Voir la critique du livre de Fernand Ouellet (Histoire économique et sociale...) par Hilda Neatby, dans Revue du Centre d'étude du Québec, no 1 (avril 1967) : 49. 
première moitié du $\mathrm{XIX}^{\circ}$ siècle, en exploitant ces prix nouveaux. Pour notre part, dans notre communication au Colloque de statistique, nous avons présenté certains indices de prix à la consommation fort simples. Cependant, il faudra manier un plus grand nombre de prix avec plus de rigueur et en utilisant des pondérations plus précises, avant d'être en mesure de comparer nos indices à ceux élaborés aux États-Unis et en Angleterre pour la même période. Il en va de même pour l'étude fascinante du niveau de vie des divers groupes sociaux selon leurs occupations et les différentes régions de la province: étude que nous avons tout juste ébauchée pour la période 1805-1812.

Dans cet article, nous nous limiterons à aligner un peu plus d'une vingtaine de séries de prix mensuels de produits importants, notamment ceux retenus par Ouellet. Elles ne veulent qu'illustrer l'évolution et la variété des prix "officiels". L'examen de ces variations peut révéler beaucoup. Cependant, derrière ces chiffres et ces courbes, c'est le mécanisme du marché et tout un arrière-plan qualitatif qu'il faut déchiffrer. ${ }^{20}$ Or une telle analyse ne peut que révéler la complexité des causalités en économie historique. A ces séries de prix, nous avons ajouté un simple indice de prix à la consommation calculé à partir des données mensuelles pour les prix du blé, des pois,

${ }^{20} \mathrm{~A}$ titre d'exemple, prenons le cas de la hausse subite et rapide puis de la stabilisation relative du prix du foin, à partir de la fin de 1808 , et la montée du prix jusque-là immuable du sable, à compter de 1809. Dans le premier cas, l'explication "bonne ou mauvaise récolte" ne suffit pas. Dans leß deux cas, en effet, l'expansion formidable des travaux de construction, surtout de fortifications, a provoqué une demande excessive de chevaux et de matériaux. Et à proximité de Québec, où le gouverneur Craig emploie un grand nombre de cultivateurs et leurs chevaux, des habitants abandonnent même la culture du blé pour celle du foin, tant celle-ci devient payante. La pratique semble bien établie en 1812: par exemple, le locataire du moulin de l'Ancienne-Lorette, Louis Déry, se plaint d'une baisse dans ses affaires car "depuis quelques années les habitants de l'ancienne Lorette au lieu d'ensemencer leurs terres en bled comme ci-devant les laissent en majeure partie en prairie pour n'en récolter que du foin, y trouvant plus d'avantage par la proximité de la ville..." (APQ, procès-verbaux des commissaires des biens des Jésuites [QBC-19], 3: 473-474). Autre exemple, la stabilité relative des prix à partir de 1810 peut s'expliquer, en partie, par l'ouverture de la route de Craig depuis Québec jusque dans les townships: le gouverneur, en effet, mentionne comment cette route canalise désormais à Québec des produits qui s'écoulaient par les Etats-Unis et stabilise - dans certains cas, fait diminuer - les prix (APC, M.G. 24, B. 3, 2:12). 
TABLEAU V

Prix courants sur le marché de Québec (1805-1812)*

1805

Blé (minot)

Avoine (minot)

Orge (minot)

Pois (minot)

Farine fine (baril)

Biscuits communs

$$
\text { ("per cwt") }
$$

Porc frais (livre)

Bouf frais (livre)

Beurre (livre)

Rhum Jamaïque (gailon)

Mélasse (gallon)

Café (livre)

Bois à chauffage (corde)

Charbon ("per chaldron")

Chandelles (livre)

Huile à lampe (gallon)

Foin (par 100 bottes de 16 livres ch.)

Chêne (pied cube)

Cerceaux $\left(12^{\prime} \times 14^{\prime}\right.$, mille $)$

Briques (mille)

Sable (charge)

Bardeaux (mille)

Pin (pied cube)

\begin{tabular}{|c|c|c|c|}
\hline Janv. & Fév. & Mars & Avril \\
\hline - & - & - & $8 /-$ \\
\hline - & - & 一 & $2 / 3$ \\
\hline - & - & - & \\
\hline - & - & - & $5 /-$ \\
\hline - & - & - & \\
\hline - & - & - & $28 /-$ \\
\hline - & & - & $5^{d}$ \\
\hline - & - & - & $5^{\mathrm{d}}$ \\
\hline & - & - & $11^{d}$ \\
\hline - & - & 一 & $6 i-$ \\
\hline - & 二 & 二 & $19^{a}$ \\
\hline E & 三 & 二 & $12 / 6$ \\
\hline - & - & - & $45 /-$ \\
\hline & - & - & $111 / 2^{d}$ \\
\hline 一 & - & - & $2 / 6$ \\
\hline - & - & 一 & $42 / 6$ \\
\hline & & - & $10^{1 / 2 d}$ \\
\hline & & $=$ & $40 /-$ \\
\hline & & - & $6^{d}$ \\
\hline & & 一 & $8 / 9$ \\
\hline & - & - & - \\
\hline
\end{tabular}

\begin{tabular}{|c|c|}
\hline Juin & Juil. \\
\hline $\begin{array}{r}7 / 6 \\
2 / 6 \\
3 / 4 \\
5 / 6 \\
47 / 6\end{array}$ & $\begin{array}{c}8 / \overline{11 / 2} \\
\overline{4 / 9} \\
51 / 3\end{array}$ \\
\hline $\begin{array}{r}27 / 6 \\
6^{d} \\
5^{d}\end{array}$ & $\frac{28 / 9}{41 / d a}$ \\
\hline $10_{1 / 2}^{0 d}$ & $131 / 2^{\mathrm{d}}$ \\
\hline & \\
\hline $\begin{array}{r}20^{d} \\
9 / 2\end{array}$ & $\begin{array}{l}22^{d} \\
9 / 10\end{array}$ \\
\hline $\begin{array}{l}30 / \\
12^{\mathrm{d}} \\
2 / 6\end{array}$ & $\begin{array}{c}35 / / \overline{1} \\
121 / d^{d} \\
2 / 9\end{array}$ \\
\hline $35 /-\overline{14^{\mathrm{d}}}$ & $35 /-\frac{1}{15^{\mathrm{d}}}$ \\
\hline 901 - & $115 /-$ \\
\hline $40 / 6^{d}$ & 二 \\
\hline$\frac{10 /-}{5^{d}}$ & $41 / 2 d$ \\
\hline
\end{tabular}

Aout

$6 /-$
$1 / 8$
$2 / 6$
$5 /-$

$40 /-$

$24 / 2$

$36^{d}$

$10^{\mathrm{d}}$

$5 / 101 / 2$

$3 / 8$
$19 d$

$8 / 4$

$30 /-$

$12^{\mathrm{d}}$
$2 / 9$

$\overline{1 /}$

£ 5.15

- $40 \%$

21/2d

Sept.

$6 / 9$

$1 / 8$

$4 / 6$

$42 / 6$

$23 / 4$

$6^{\mathrm{d}}$
$4^{\mathrm{d}}$
$1^{\mathrm{d}}$

$4 / 91 / 2$

$3 / 4$

$18^{\mathrm{d}}$
$8 / 4$

$30 \%$

$11^{d}$
$2 / 9$

$35 /-$

$40 /-$

$2 \overline{3 / 4}$
Oct. Nov.

Déc.

$6 / 9 \quad 6 / 8$

$2 /-\quad 2 /-$

$5 /-\quad 5 / 6$

$45 /$ - $42 / 6$

$5 / 10$

$2 /$ -

5/-

$42 / 6$

$25 /-$

$23 / 4$

$\overline{31 / 2} \mathrm{~d}$

$101 / 2 \mathrm{~d}$

$5 /$ -

$3 / 3$

$19^{d}$

$9 / 10 \quad 12 / 6$

$35 /-40 /-$

$12^{d} \quad 40$

$2 / 9 \quad 2 / 9$

$2 / 9$

$40 /-\quad 40 /-$

$12^{\mathrm{d}}$

$f 6.5$

$£ 6.5$

$40 /-$

£ 6.5

$40 /-$

£ 6.10

$6^{\mathrm{d}}$

$\overline{21 / 2 d}$

* Source: rapports mensuels du clerc du marché transmis à Londres par l'administrateur de la colonie, à compter d'avril 1805 (APC, M.G.11, série Q). L'unité monétaire est la livre anglaise, cours de la colonie. Les quelques cotes en monnaie sterling ou en dollars ont été converties en monnaie courante selon les taux en usage à l'époque: $£ 1$ courant $=9 / 10$ de 1 sterling; $\$ 1$. $=$ une piastre espagnole $=4 / 6$ (4 shillings 6 deniers) sterling ou 5/- (5 shillings) courant. Il y a douze deniers au shilling, vingt shillings à la livre. Dans nos calculs de transposition, nous n'avons omis que les fractions de denier. Dans certains cas, confrontés à plusieurs prix à l'intérieur d'un même mois, nous avons calculé un prix moyen. L'absence de prix pour tel produit, à tel moment, peut découler d'un ou de plusieurs facteurs: ignorance de ce prix; trop grandes fluctuations du marché; déficience dans l'offre ou la demande 
1806

Blé (minot)

Avoine (minot)

Orge (minot)

Pois (minot)

Farine fine (baril)

Biscuits commūns ("per cwt")

Porc frais (livre)

Bœuf frais (livre)

Beurre (livre)

Mélasse (gallon)

Café (livre)

Bois à chauffage (corde)

Charbon ("per chaldron")

Chandelles (livre)

Huile à lampe (gallon) 16 livres ch.)

Chêne (pied cube)

Cerceaux (12' x 14', mille)

Briques (mille)

Sable (charge)

Bardeaux (mille)

Pin (pied cube)
Rhum Jamaïque (gallon)

Foin (par 100 bottes de

\begin{tabular}{|c|c|c|c|c|c|c|c|c|c|c|c|}
\hline Janv. & Fév. & Mars & Avril & Mai & Juin & Juil. & Août & Sept. & Oct. & Nov. & Déc. \\
\hline $7 / 1 / 2$ & $7 /-$ & $6 / 6$ & $6 / 8$ & $7 / 1$ & $7 / 6$ & $6 / 9$ & $6 / 9$ & $6 /-$ & $5 / 9$ & $5 /-$ & $5 / 6$ \\
\hline $2 / 11 / 2$ & $1 / 8$ & $2 /-$ & $2 /-$ & $2 /-$ & $2 /-$ & $2 /-$ & $2 / 6$ & $3 /-$ & $2 / 6$ & $2 /-$ & $1 / 8$ \\
\hline $2 / 9$ & $3 /-$ & $3 /-$ & $3 /-$ & $2 / 9$ & $2 / 6$ & $2 / 6$ & $3 /-$ & $3 / 4$ & $3 /-$ & $3 /-$ & - \\
\hline $4 / 6$ & $5 /-$ & $5 /-$ & $5 /-$ & $4 / 9$ & $4 / 71 / 2$ & $4 / 3$ & $5 /-$ & $4 / 9$ & $4 / 6$ & $4 / 6$ & $5 /-$ \\
\hline $47 / 6$ & $40 /-$ & $40 /-$ & $40 /-$ & $40 /-$ & $45 /-$ & $45 /-$ & $40 /-$ & $37 / 6$ & $37 / 6$ & - & - \\
\hline $27 / 6$ & - & - & $20 /-$ & $21 / 6$ & $25 /-$ & $23 / 4$ & $23 / 4$ & $22 / 6$ & $22 / 6$ & - & - \\
\hline - & $6^{d}$ & $51 / 2 d$ & $51 / 2 d$ & $51 / 2 d$ & $51 / 2^{d}$ & $6^{d}$ & - & $61 / 2^{d}$ & $7 \mathrm{~d}$ & $6^{\mathrm{d}}$ & $51 / 2^{d}$ \\
\hline - & $4^{d}$ & $4^{d}$ & $4^{d}$ & $41 / 2 d$ & $41 / 2 d$ & $41 / 2 d$ & $4^{d}$ & $4^{d}$ & $4^{d}$ & $31 / 2^{d}$ & $31 / 2^{d}$ \\
\hline $101 / 2^{d}$ & $9^{d}$ & $10^{d}$ & $10^{d}$ & $101 / 2 d$ & $101 / 2 d$ & $10^{d}$ & $10^{d}$ & $10^{d}$ & $10^{d}$ & $10^{d}$ & $10^{d}$ \\
\hline $4 / 101 / 2$ & $5 /-$ & $5 /-$ & $5 /-$ & $5 /-$ & $4 / 101 / 2$ & $5 /-$ & $4 / 9$ & $4 / 9$ & $4 / 9$ & $5 /-$ & $4 / 101 / 2$ \\
\hline $3 / 31 / 2$ & $3 / 9$ & $4 /-$ & $4 /-$ & $3 / 9$ & $3 /-$ & $3 /-$ & $3 /-$ & $3 /-$ & $3 /-$ & $3 / 6$ & $3 /-$ \\
\hline $201 / 2 d$ & $20^{d}$ & $20^{d}$ & $20^{d}$ & $20^{d}$ & $1 / 7$ & $20^{d}$ & $19^{d}$ & $18^{d}$ & $18^{d}$ & $20^{\mathrm{d}}$ & - \\
\hline $12 / 1$ & $12 / 6$ & $12 / 6$ & $12 / 6$ & $13 /-$ & $7 / 11$ & $7 / 11$ & $8 / 4$ & $7 / 11$ & $10 /-$ & $10 /-$ & $10 /-$ \\
\hline $40 /-$ & - & $36 /-$ & $36 /-$ & $38 /-$ & $30 /-$ & $30 /-$ & $30 /-$ & $30 /-$ & $30 /-$ & $26 /-$ & $30 /-$ \\
\hline $13^{d}$ & $12^{d}$ & $11^{d}$ & $11^{d}$ & $11^{d}$ & $12^{d}$ & $11^{d}$ & $11^{d}$ & $12^{d}$ & $12^{d}$ & $12^{d}$ & $12^{\mathrm{d}}$ \\
\hline $2 / 9$ & $2 / 6$ & $2 / 9$ & $2 / 9$ & $2 / 9$ & $2 / 6$ & $2 / 9$ & $2 / 9$ & $2 / 9$ & $2 / 9$ & $2 / 9$ & $2 / 9$ \\
\hline $30 /-$ & $25 /-$ & $30 /-$ & $40 / 6$ & $35 /-$ & $40 /-$ & $40 /-$ & $40 \%-$ & $40 /-$ & $40 /-$ & $40 /-$ & $40 /-$ \\
\hline $15^{d}$ & - & - & - & - & $151 / 2 d$ & $151 / 2 d$ & $15^{d}$ & $16^{\mathrm{d}}$ & $19^{d}$ & - & - \\
\hline $115 /-$ & $110 /-$ & $128 /-$ & $120 /-$ & $120 /-$ & $110 /-$ & $110 /-$ & $120 /-$ & $100 /-$ & $120 /-$ & - & - \\
\hline - & - & - & - & - & $50 /-$ & $50 /-$ & $50 /-$ & $50 /-$ & $40 /-$ & - & - \\
\hline - & - & - & - & - & $6^{d}$ & $6^{d}$ & $6^{d}$ & $6^{d}$ & $71 / 2^{d}$ & - & - \\
\hline - & $7 / 6$ & $10 /-$ & $10 /-$ & $12 / 6$ & $10 /-$ & $10 /-$ & $10 /-$ & $10 /-$ & $10 /-$ & - & - \\
\hline $31 / 2 d$ & - & - & - & - & $51 / 2 d$ & $51 / 2 d$ & $5^{d}$ & $6^{d}$ & $6^{d}$ & - & - \\
\hline
\end{tabular}

équivaloir aux premières; etc. 
1807

Blé (minot)

Avoine (minot)

Orge (minot)

Pois (minot)

Farine fine (baril)

Biscuits communs ("per cwt")

Porc frais (livre)

Bœuf frais (livre)

Beurre (livre)

Rhum Jamaïque (gallon)

Mélasse (gallon)

Café (livre)

Bois à chauffage (corde)

Charbon ("per chaldron")

Chandelles (livre)

Huile à lampe (gallon)

Foin (par 100 bottes de 16 livres ch.)

Chêne (pied cube)

Cerceaux (12' x 14', mille)

Briques (mille)

Sable (charge)

Bardeaux (mille)

Pin (pied cube)
Janv. Fév. Mars Avril Mai Juin Juil. Août Sept. Oct. Nov. Déc.

$6 /$ - $6 / 4 \quad 6 / 4 \quad 6 / 8 \quad-\quad 7 / 9 \quad 7 / 4 \quad 7 /$ - $\quad 6 \quad-\quad 6 / 8 \quad 6 / 8 \quad 6 /$ -

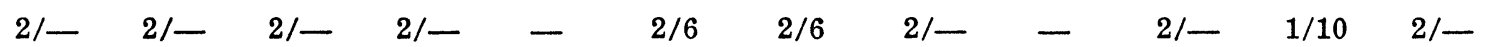

$3 /-3 /-3 /-3 /-\quad-3 /-3 /-3 /-\quad-\quad 3 /-3 /-3 / 6$

$5 /$ - 5/- 5/- 5/- $\quad$ - $5 /$ - $5 / 6 \quad 5 / 9 \quad-\quad 4 / 101 / 24 / 101 / 2 \quad 4 / 9$

$35 /$ - $39 / 10^{1 / 2} 39 / 101 / 2 \quad 40 /$ - $\quad-35 / 3 \quad 32 / 6 \quad 32 / 6 \quad-\quad 32 / 6 \quad 32 / 6 \quad 32 / 6$

- $20 /$ - 20/一 $22 / 6 \quad-21 /$ - 20/6 20/一 $-20 /$ - 20/- $18 /$ -

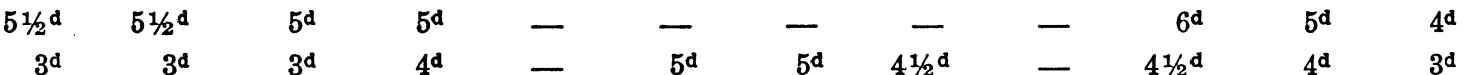

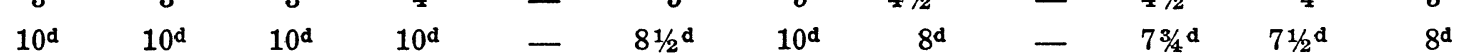

$4 / 71 / 2 \quad 4 / 71 / 2 \quad 4 / 71 / 2 \quad 4 / 101 / 2 \quad-\quad 5 /$ - $5 /$ - $6 /$ - $\quad$ - $5 / 101 / 26 / 11 / 26 /$ -

$3 /$ - $3 /$ - $3 /$ - $3 /$ - $\quad-\quad 3 / 6 \quad 3 / 6 \quad 4 / 6 \quad-\quad \begin{array}{llllll}4 / 3 & 4 / 6 & 5 / \text { - }\end{array}$

$\begin{array}{llllllllllll}19^{\mathrm{d}} & 19^{\mathrm{d}} & 18^{\mathrm{d}} & 19^{\mathrm{d}} & - & 20^{\mathrm{d}} & 20^{\mathrm{d}} & 24^{\mathrm{d}} & - & 22^{\mathrm{d}} & 22^{\mathrm{d}} & 24^{\mathrm{d}}\end{array}$

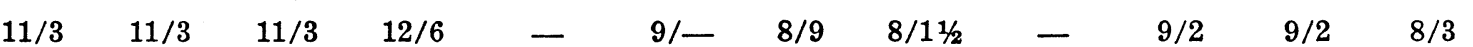

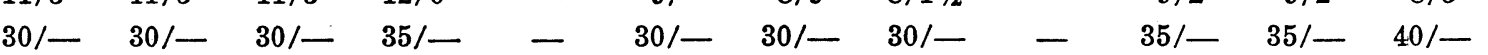

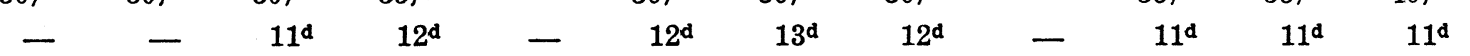

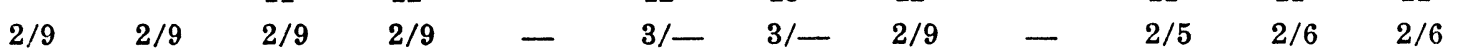

$40 /$ - $40 /$ - $40 /$ - $40 /$ - $-40 /$ - $35 /$ - $35 /$ - $-35 /$ - $35 /$ - $30 /$ -

$-\quad-\quad-\quad-\quad-\quad-\quad 13^{\mathrm{d}} 13^{\mathrm{d}} 13^{\mathrm{d}} \quad-\quad \begin{array}{cccc}14^{\mathrm{d}} & 14^{\mathrm{d}} & 14^{\mathrm{d}}\end{array}$

- - - - - $100 /-100 /-100 /-\quad-100 /-90 /-90 /-$

- $-\quad-\quad-$

$\begin{array}{lllll}- & - & - & - & - \\ - & - & - & - & -\end{array}$
-

$-$
=

$$
\begin{array}{ccc}
- & - & - \\
- & 6^{\mathrm{d}} & - \\
- & 10 /- & -
\end{array}
$$

6

$6^{d}$

- -1 -
$7 \mathrm{~d}$

$534^{d}$ 


\section{8}

Blé (minot)

Avoine (minot)

Orge (minot)

Pois (minot)

Farine fine (baril)

Biscuits communs ("per cwt")

Porc frais (livre)

Bœuf frais (livre)

Beurre (livre)

Rhum Jamaĩque (gallon)

Mélasse (gallon)

Café (livre)

Bois à chauffage (corde)

Charbon ("per chaldron")

Chandelles (livre)

Huile à lampe (gallon)

Foin (par 100 bottes de 16 livres ch.)

Chêne (pied cube)

Cerceaux (12' x 14', mille)

Briques (mille)

Sable (charge)

Bardeaux (mille)

Pin (pied cube)

\begin{tabular}{|c|c|c|c|c|c|c|c|c|c|c|c|}
\hline Janv. & Fév. & Mars & Avril & Mai & Juin & Juil. & Août & Sept. & Oct. & Nov. & Déc. \\
\hline $5 / 6$ & $5 / 6$ & $7 / 6$ & $7 / 6$ & $7 / 9$ & $8 /-$ & $3 /$ Z** $^{* *}$ & $8 / 6$ & $7 / 9$ & $1 /$ - $^{* *}$ & $7 /-$ & - \\
\hline $1 / 6$ & $1 / 8$ & $20^{d}$ & $2 /-$ & $2 /-$ & $2 /-$ & $2 / 6$ & $3 /-$ & $3 /-$ & $3 /-$ & $3 /-$ & $3 /-$ \\
\hline $2 / 9$ & $3 /-$ & $3 /-$ & $2 / 9$ & $2 / 9$ & $2 / 9$ & $2 / 6$ & - & - & $3 / 4$ & $3 /-$ & - \\
\hline $4 / 6$ & $4 / 9$ & $5 / 6$ & $5 / 6$ & $6 /-$ & $6 / 9$ & $6 / 9$ & $8 /-$ & $6 /-$ & $5 / 6$ & $6 /-$ & - \\
\hline $32 / 6$ & $32 / 6$ & $32 / 6$ & $35 /-$ & $37 / 6$ & $40 /-$ & $47 / 6$ & $48 / 9$ & $50 /-$ & $45 /-$ & $45 /-$ & $45 /-$ \\
\hline $20 /-$ & $10 / 6$ & $23 / 4$ & $23 / 4$ & $23 / 4$ & $25 /-$ & $25 /-$ & $25 /-$ & $25 /-$ & $25 /-$ & $25 i-$ & $25 /-$ \\
\hline $\mathbf{5}^{\mathbf{d}}$ & $5^{d}$ & $5^{d}$ & $5^{d}$ & $5^{d}$ & $\mathbf{5}^{\mathbf{d}}$ & - & - & - & $5^{d}$ & $5^{d}$ & $5^{d}$ \\
\hline $3^{d}$ & $31 / 2 d$ & $4^{\mathrm{d}}$ & $4^{d}$ & $41 / 2^{d}$ & $41 / 2^{d}$ & $5^{d}$ & $5^{d}$ & $5^{d}$ & $4^{d}$ & $4^{\mathrm{d}}$ & $31 / 4 d^{d}$ \\
\hline- & $9^{d}$ & $78 / 4^{d}$ & $71 / 2^{d}$ & $8^{d}$ & $8^{d}$ & $8^{d}$ & $10^{d}$ & $10^{d}$ & $9^{d}$ & $10^{d}$ & $10^{d}$ \\
\hline $6 / 3$ & $6 / 3$ & $6 /-$ & $6 /-$ & $5 / 9$ & $4 / 71 / 2$ & $5 / 9$ & $4 / 10^{1 / 2}$ & $5 / 6$ & $7 /-$ & $7 / 9$ & $9 / 3$ \\
\hline $4 /-$ & $4 /-$ & $3 / 6$ & $3 / 4$ & $3 /-$ & $2 / 9$ & $2 / 6$ & $3 /-$ & $3 /-$ & $3 / 6$ & $3 / 6$ & $3 /-$ \\
\hline $22^{d}$ & $22^{\mathrm{d}}$ & $24^{\mathrm{d}}$ & $24^{\mathrm{d}}$ & $20^{d}$ & $18^{d}$ & $18^{\mathrm{d}}$ & $18^{a}$ & $161 / 2^{d}$ & $161 / 2^{a}$ & $161 / 2 d$ & $16^{\mathrm{d}}$ \\
\hline $11 / 3$ & $12 / 6$ & $12 / 6$ & $12 / 6$ & $10 /-$ & $10 /-$ & $10 /-$ & $10 /-$ & $12 / 6$ & $20 /-$ & $16 / 8$ & $18 / 4$ \\
\hline $40 \%$ & $40 /-$ & $40 /-$ & $40 /-$ & $30 /-$ & $30 /-$ & $25 /-$ & $30 /-$ & $35 /-$ & $40 /-$ & $40 /-$ & $45 /-$ \\
\hline $11^{d}$ & $11^{d}$ & $11^{d}$ & $11^{d}$ & $11^{\mathrm{d}}$ & $11^{d}$ & $11^{d}$ & $12^{d}$ & $111 / 2^{d}$ & $12^{d}$ & $11^{d}$ & $11^{d}$ \\
\hline $2 / 9$ & $2 / 6$ & $2 / 6$ & $2 / 6$ & $2 / 6$ & $2 / 6$ & $2 / 6$ & $2 / 6$ & $2 / 6$ & $2 / 6$ & $2 / 6$ & $2 /-$ \\
\hline $32 / 6$ & $35 /-$ & $40 /-$ & $40 /-$ & $40 /-$ & $40 /-$ & $40 \%-$ & $40 /-$ & $40 /-$ & $50 /-$ & $70 /-$ & $55 /-$ \\
\hline- & - & - & - & $18^{d}$ & $22^{d}$ & $2 /-$ & $22^{d}$ & $22^{d}$ & $22^{d}$ & $20^{d}$ & - \\
\hline - & - & - & - & $£ 6$ & $£ 6$ & $£ 6$ & $£ 6$ & $£ 6$ & $£ 6$ & $£ 5$ & - \\
\hline- & - & - & - & $50 /-$ & $50 /-$ & $50 /-$ & $45 /-$ & - & $45 /-$ & $40 /-$ & $50 /-$ \\
\hline- & - & - & - & $6^{\mathrm{d}}$ & - & $6^{d}$ & $6^{d}$ & - & $6^{d}$ & $6^{d}$ & - \\
\hline - & - & - & - & $12 / 6$ & - & $15 /-$ & $12 / 6$ & - & $12 / 6$ & $12 / 6$ & - \\
\hline- & - & - & - & $9^{d}$ & $9^{d}$ & $17^{d}$ & $121 / 2^{d}$ & $10^{d}$ & $91 / 2^{d}$ & $8^{d}$ & - \\
\hline
\end{tabular}

** Erreur de transcription du commis ? 


\section{9}

Blé (minot)

Avoine (minot)

Orge (minot)

Pois (minot)

Farine fine (baril)

Biscuits communs ("per cwt")

Porc frais (livre)

Bœuf frais (livre)

Beurre (livre)

Rhum Jamaĩque (gallon)

Mélasse (gallon)

Café (livre)

Bois à chauffage (corde)

Charbon ("per chaldron")

Chandelles (livre)

Huile à lampe (gallon)

Foin (par 100 bottes de 16 livres ch.)

Chêne (pied cube)

Cerceaux (12' x 14', mille)

Briques (mille)

Sable (charge)

Bardeaux (mille)

Pin (pied cube)
Janv. Fév. Mars Avril Mai Juin Juil. Août Sept. Oct. Nov. Déc.

\begin{tabular}{|c|c|c|c|c|c|c|c|c|c|c|c|}
\hline $5 / 4$ & $8 / 4$ & $8 / 4$ & $81-$ & $8 /-$ & - & $7 / 6$ & $7 /-$ & $7 / 6$ & $8 / 6$ & $8 /-$ & $8 / 6$ \\
\hline $2 / 6$ & $2 / \theta$ & $3 /-$ & $3 / 6$ & $3 /-$ & - & $3 /-$ & $3 / 6$ & $3 /-$ & $3 / 6$ & $3 / 6$ & $3 /-$ \\
\hline $3 / 4$ & - & - & - & - & - & - & - & - & $3 / 4$ & - & $3 / 4$ \\
\hline $7 / 6$ & $7 / 6$ & $7 / 9$ & $81-$ & $8 /-$ & - & $7 /-$ & $7 /-$ & $7 / 6$ & $8 /-$ & $8 /-$ & $8 /-$ \\
\hline $45 /-$ & $47 / 6$ & $50 /-$ & $47 / 6$ & $47 / 6$ & - & $42 / 6$ & $42 / 6$ & $45 /-$ & $50 /-$ & $55 /-$ & $55 /-$ \\
\hline $27 / 6$ & $28 /-$ & $27 / 6$ & $28 /-$ & $27 / 6$ & - & $25 /-$ & $25 /-$ & $26 /-$ & $27 / 6$ & $32 / 6$ & - \\
\hline $41 / 2^{d}$ & $43 / 4^{d}$ & $5^{d}$ & $6^{d}$ & $7 d$ & - & - & $7^{d}$ & $6^{\mathrm{d}}$ & $6^{d}$ & $6^{\mathrm{d}}$ & - \\
\hline $21 / 2^{d}$ & $\mathbf{3}^{\mathbf{d}}$ & $4^{d}$ & $6^{d}$ & $7 \mathrm{~d}$ & - & $71 / 2^{d}$ & $61 / 2 d$ & $4^{d}$ & $4^{a}$ & $4^{d}$ & - \\
\hline $10^{\mathrm{d}}$ & $9 d$ & $11^{\mathrm{d}}$ & $11^{d}$ & $10 \mathrm{~d}$ & - & $12^{d}$ & $12^{d}$ & $10^{d}$ & $12^{d}$ & $12^{d}$ & - \\
\hline $8 / 6$ & $8 / 9$ & $8 / 9$ & $8 /-$ & $6 / 9$ & - & $6 /-$ & $6 / 8$ & $7 /-$ & $7 / 9$ & $8 /-$ & - \\
\hline $4 /-$ & $4 / 6$ & $3 / 4$ & $4 /-$ & $3 / 4$ & - & $3 /-$ & $3 /-$ & $3 /-$ & $3 / 6$ & $3 /-$ & - \\
\hline $10^{d}$ & $15^{d}$ & $16^{\mathrm{d}}$ & $16^{d}$ & $16^{d}$ & - & $15^{d}$ & $15^{d}$ & $16^{d}$ & $18^{\mathrm{d}}$ & $16^{\mathrm{d}}$ & - \\
\hline $20 /-$ & $20 /-$ & $22 / 6$ & $20 /-$ & - & - & $15 /-$ & $16 / 8$ & $17 / 6$ & $20 /-$ & $22 / 6$ & - \\
\hline $50 /-$ & $50 /-$ & $45 /-$ & $50 /-$ & $35 /-$ & - & $281 / 2 d$ & $35 / 9$ & $37 / 6$ & $47 / 6$ & $45 /-$ & - \\
\hline $12^{d}$ & $12^{\mathrm{d}}$ & $12^{\mathrm{d}}$ & $12^{\mathrm{d}}$ & $12^{\mathrm{d}}$ & - & $13^{d}$ & $13^{d}$ & $13^{d}$ & $14^{\mathrm{d}}$ & $14^{\mathrm{d}}$ & - \\
\hline $2 / 6$ & $2 / 6$ & $2 / 9$ & $3 /-$ & $2 / 6$ & - & $2 / 6$ & $2 / 6$ & $2 / 9$ & $3 /-$ & $3 /-$ & - \\
\hline $60 /-$ & $75 /-$ & $100 /-$ & - & $90 /-$ & - & $80 /-$ & $70 /-$ & $75 /-$ & $80 /-$ & $90 /-$ & - \\
\hline $16^{\mathrm{d}}$ & $18^{d}$ & $2 /-$ & $2 /-$ & $2 /-$ & - & $20^{d}$ & $191 / 2^{d}$ & $19^{d}$ & $19^{d}$ & $18^{d}$ & - \\
\hline- & $110 /-$ & $£ 6$ & $100 /-$ & $100 /-$ & - & $100 /-$ & $90 /-$ & $£ 6$ & $£ 6$ & $£ 6$ & - \\
\hline $50 /-$ & $50 /-$ & $50 /-$ & $50 /-$ & $50 /-$ & - & $50 /-$ & $50 /-$ & $50 /-$ & $50 /-$ & $50 /-$ & - \\
\hline- & - & - & $6^{d}$ & $6^{\mathrm{d}}$ & - & $6^{d}$ & $6^{d}$ & $6^{d}$ & $6^{\mathrm{d}}$ & $1 /-$ & - \\
\hline $12 / 6$ & $12 / 6$ & - & $12 / 6$ & $12 / 6$ & - & $12 / 6$ & $12 / 6$ & $12 / 6$ & $15 /-$ & $15 /-$ & - \\
\hline $71 / 2^{d}$ & $63 / 4^{d}$ & $121 / 2^{d}$ & $12^{\mathrm{d}}$ & $11^{d}$ & - & $6^{d}$ & $6^{\mathrm{d}}$ & $7^{\mathrm{d}}$ & $6^{d}$ & $6^{d}$ & - \\
\hline
\end{tabular}


1810

Blé (minot)

Avoine (minot)

Orge (minot)

Pois (minot)

Farine fine (baril)

Biscuits communs ("per cwt")

Porc frais (livre)

Bœuf frais (livre)

Beurre (livre)

Rhum Jamaïque (gallon)

Mélasse (gallon)

Café (livre)

Bois à chauffage (corde)

Charbon ("per chaldron")

Chandelles (livre)

Huile à lampe (gallon)

Foin (par 100 bottes de 16 livres ch.)

Chêne (pied cube)

Cerceaux (12' x 14', mille)

Briques (mille)

Sable (charge)

Bardeaux (mille)

Pin (pied cube)

\begin{tabular}{|c|c|c|c|c|c|c|c|c|c|c|}
\hline Janv. & Fév. & Mars & Avril & Mai & Juin & Juil. & Août & Sept. & Oct. & Nov. \\
\hline $8 / 4$ & $8 / 51 / 2$ & $8 / 6$ & $8 / 6$ & $8 / 4$ & $8 / 6$ & $8 / 6$ & $10 / 7$ & $10 / 7$ & $10 / 7$ & $11 /-$ \\
\hline $2 / 9$ & $2 / 3$ & $2 / 6$ & $2 / 6$ & $8 /-$ & $3 /-$ & $3 /-$ & $3 / 6$ & $4 /-$ & $3 / 6$ & $3 / 6$ \\
\hline- & - & - & - & - & - & - & - & $3 / 6$ & - & $3 /-$ \\
\hline $7 / 6$ & $6 / 6$ & $6 / 6$ & $6 / 6$ & $7 /-$ & $7 / 6$ & $7 /-$ & $7 / 6$ & $7 / 6$ & $8 / 6$ & $8 / 6$ \\
\hline $55 /-$ & $50 /-$ & $50 /-$ & $50 /-$ & $52 / 6$ & $52 / 6$ & $52 / 6$ & $55 /-$ & $60 /-$ & $60 /-$ & $65 /-$ \\
\hline $30 /-$ & $30 /-$ & $30 /-$ & $30 /-$ & $30 /-$ & $28 /-$ & $27 / 6$ & $28 /-$ & $32 / 6$ & $30 /-$ & $35 /-$ \\
\hline $91 / 2^{d}$ & $9^{d}$ & $9^{d}$ & $8^{d}$ & - & - & - & $8^{d}$ & $10^{d}$ & $9^{d}$ & $9^{d}$ \\
\hline $6^{d}$ & $6^{d}$ & $6^{d}$ & $6^{d}$ & $9^{d}$ & $71 / 2^{d}$ & $61 / 2^{d}$ & $63 / 4 d$ & $6^{d}$ & $6^{d}$ & $5^{d}$ \\
\hline $11^{d}$ & $12^{\mathrm{d}}$ & $12^{d}$ & $12^{d}$ & $12^{\mathrm{d}}$ & $12^{\mathrm{d}}$ & $12^{\mathrm{d}}$ & $12^{d}$ & $12^{d}$ & $12^{d}$ & $12^{d}$ \\
\hline $8 /-$ & $8 / 6$ & $8 /-$ & $7 / 9$ & $6 /-$ & $6 /-$ & $6 / 3$ & $6 / 9$ & $6 / 6$ & $6 / 9$ & $6 / 6$ \\
\hline $3 / 6$ & $3 /-$ & $3 / 6$ & $3 / 6$ & $3 /-$ & $3 / 6$ & $4 /-$ & $3 / 10^{1 / 2}$ & $4 / 6$ & $4 /-$ & $4 /-$ \\
\hline $15^{\mathrm{d}}$ & $18^{d}$ & $16^{d}$ & $16^{d}$ & $18^{d}$ & $15^{d}$ & $15^{\mathrm{d}}$ & $16^{d}$ & $18^{\mathrm{d}}$ & $16^{d}$ & $18^{d}$ \\
\hline $20 /-$ & $20 /-$ & $20 /-$ & $20 /-$ & $20 /-$ & $15 /-$ & $16 / 8$ & $15 /-$ & $16 / 3$ & $17 / 6$ & $17 / 6$ \\
\hline $50 /-$ & - & - & - & $30 /-$ & $25 /-$ & $25 /-$ & $25 /-$ & $25 /-$ & $30 /-$ & $40 /-$ \\
\hline $14^{\mathrm{d}}$ & $14^{\mathrm{d}}$ & $14^{\mathrm{d}}$ & $14^{\mathrm{d}}$ & $14^{d}$ & $13^{d}$ & $13^{d}$ & $12^{\mathrm{d}}$ & $13^{d}$ & $15^{\mathrm{d}}$ & $15^{\mathrm{d}}$ \\
\hline $3 /-$ & $3 /-$ & $3 /-$ & $3 /-$ & $2 / 9$ & $3 / 4$ & $4 /-$ & $4 /-$ & $4 /-$ & $4 /-$ & $4 /-$ \\
\hline $90 /-$ & $75 /-$ & $75 /-$ & $75 /-$ & $70 /-$ & $75 /-$ & $80 /-$ & $80 /-$ & $80 \%-$ & - & $90 /-$ \\
\hline- & - & - & $20^{d}$ & $2 /-$ & $2 / 11 / 2$ & $2 / 41 / 2$ & $2 /-$ & $2 / 3$ & $2 / 6$ & $2 / 9$ \\
\hline- & - & - & $£ 5.10$ & $£ 6$ & $£ 6.10$ & $115 /-$ & $£ 6$ & $£ 6$ & $£ 6$ & $\mathcal{L} 5.10$ \\
\hline- & - & - & $50 /-$ & $50 /-$ & $50 /-$ & $50 /-$ & $50 /-$ & $50 /-$ & $50 /-$ & $50 /-$ \\
\hline- & - & - & $12^{d}$ & $9^{d}$ & $6^{d}$ & $71 / 2 d$ & $9^{d}$ & $9^{d}$ & $71 / 2^{d}$ & $9^{d}$ \\
\hline- & - & - & $12 / 6$ & $12 / 6$ & $12 / 6$ & $12 / 6$ & $12 / 6$ & $12 / 6$ & $12 / 6$ & $10 /-$ \\
\hline- & - & - & $6^{\mathrm{d}}$ & $1^{d}$ & $61 / 4^{d}$ & $51 / 2^{d}$ & $41 / 2^{d}$ & $51 / 2 d$ & $51 / 2^{d}$ & $41 / 2^{d}$ \\
\hline
\end{tabular}


1811

Blé (minot)

Avoine (minot)

Orge (minot)

Pois (minot)

Farine fine (baril)

Biscuits communs

$$
\text { ("per cwt") }
$$

Porc frais (livre)

Bœuf frais (livre)

Beurre (livre)

Rhum Jamaïque (gallon)

Mélasse (gallon)

Café (livre)

Bois à chauffage (corde)

Charbon ("per chaldron")

Chandelles (livre)

Huile à lampe (gallon)

Foin (par 100 bottes de 16 livres ch.)

Chêne (pied cube)

Cerceaux (12' x 14', mille)

Briques (mille)

Sable (charge)

Bardeaux (mille)

Pin (pied cube)
Janv. Fév. Mars Avril Mai Juin Juil. Août Sept. Oct. Nov. Déc.

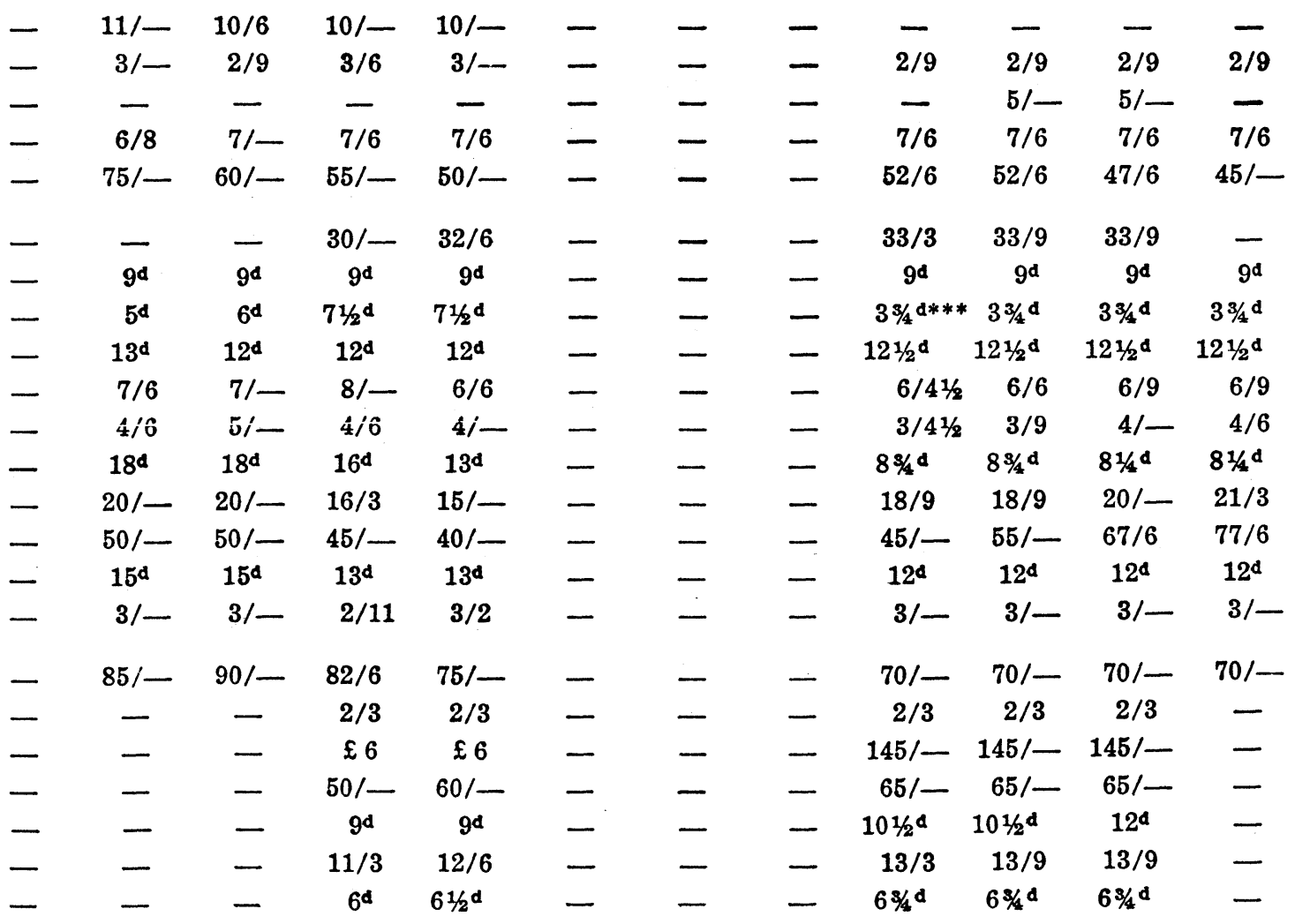

** A compter de septembre 1811, l'unité du bouf frais devient le "cwt" au lieu de la livre. Il a donc fallu calculer le prix de la livre en attribuant au "cwt" sa valeur de 112 livres. 


\section{2}

Blé (minot)

Avoine (minot)

Orge (minot)

Pois (minot)

Farine fine (baril)

Biscuits communs ("per cwt")

Porc frais (livre)

Bœuf frais (livre)

Beurre (livre)

Rhum Jamaĩque (gallon)

Mélasse (gallon)

Café (livre)

Bois à chauffage (corde)

Charbon ("per chaldron")

Chandelles (livre)

Huile à lampe (gallon)

Foin (par 100 bottes de 16 livres ch.)

Chêne (pied cube)

Cerceaux (12' x 14', mille)

Briques (mille)

Sable (charge)

Bardeaux (mille)

Pin (pied cube)

\begin{tabular}{|c|c|c|c|c|c|c|c|c|c|c|c|}
\hline $\begin{array}{r}\text { Janv. } \\
6 / 10\end{array}$ & $\begin{array}{l}\text { Fév. } \\
6 / 10\end{array}$ & $\begin{array}{c}\text { Mars } \\
7 / 91 / 2\end{array}$ & $\begin{array}{c}\text { Avril } \\
8 / 4\end{array}$ & $\begin{array}{l}\text { Mai } \\
9 / 9\end{array}$ & $\begin{array}{c}\text { Juin } \\
9 / 9\end{array}$ & $\begin{array}{l}\text { Juil. } \\
9 / 6\end{array}$ & $\begin{array}{l}\text { Août } \\
10 /-\end{array}$ & $\begin{array}{l}\text { Sept. } \\
10 /-\end{array}$ & $\begin{array}{l}\text { Oct. } \\
10 /-\end{array}$ & $\begin{array}{l}\text { Nov. } \\
10 i-\end{array}$ & $\begin{array}{c}\text { Déc. } \\
12 /-\end{array}$ \\
\hline $2 / 6$ & $2 / 6$ & $2 / 6$ & $2 / 9$ & $3 / 3$ & $3 /-$ & $2 / 71 / 8$ & $2 / 101 / 2$ & $3 / 9$ & $3 / 9$ & $3 /-$ & $3 /-$ \\
\hline- & - & - & - & - & - & - & - & - & - & $3 / 8$ & - \\
\hline $7 / 1$ & $7 / 1$ & $7 / 1$ & $7 / 1$ & $6 / 8$ & $6 / 10$ & $6 / 9$ & $7 / 1$ & $7 /-$ & $7 /-$ & $7 /-$ & $8 / 9$ \\
\hline $45 /-$ & $45 /-$ & $50 /-$ & $50 /-$ & $51 / 3$ & $52 / 6$ & $55 /-$ & $55 /-$ & $57 / 6$ & $60 /-$ & $65 /-$ & $80 /-$ \\
\hline - & - & - & $28 / 9$ & $31 / 3$ & $31 / 3$ & $31 / 3$ & $32 / 6$ & $32 /-$ & $31 / 3$ & $31 / 3$ & $31 / 3$ \\
\hline $9 d$ & $9^{d}$ & $9 d$ & $9^{d}$ & $9^{d}$ & - & - & $9 d$ & $9^{d}$ & $9^{d}$ & $9^{d}$ & $8^{d}$ \\
\hline $3 \% / 4 d$ & $33 / 4 d$ & $3 z / 4 d$ & $48 / 4 d$ & $4 \% / 2 d$ & $5^{d}$ & $5^{d}$ & $5^{d}$ & $5^{d}$ & $5^{d}$ & $5^{d}$ & $4^{\mathrm{d}}$ \\
\hline $121 / 2^{d}$ & $121 / 2^{d}$ & $121 / 2^{d}$ & $121 / 2^{d}$ & $121 / 2 d$ & $12^{d}$ & $12^{d}$ & $12^{\mathrm{d}}$ & $12^{d}$ & $12^{\mathrm{d}}$ & $12^{d}$ & $12^{d}$ \\
\hline $6 / 9$ & $6 / 9$ & $6 / 9$ & $6 / 6$ & $5 / 9$ & $5 / 9$ & $5 /-$ & $5 / 3$ & $5 / 41 / 2$ & $5 / 71 / 2$ & $5 / 101 / 2$ & $6 / 3$ \\
\hline $4 /-$ & $3 / 9$ & $3 / 9$ & $3 / 6$ & $2 / 101 / 2$ & $2 / 6$ & $2 / 2$ & $2 / 9$ & $2 / 9$ & $2 / 9$ & $2 / 9$ & $2 / 9$ \\
\hline $81 / 4^{d}$ & $71 / 2 d$ & $7^{\mathfrak{d}}$ & $7 d$ & $6^{\mathrm{d}}$ & $6^{d}$ & $6^{d}$ & $61 / 2 d$ & $7 d$ & $71 / 2 d$ & $81 / 2^{d}$ & $9^{d}$ \\
\hline $23 / 9$ & $23 / 6$ & $23 / 9$ & $30 /-$ & $25 /-$ & - & $14 / 7$ & $13 / 9$ & $14 /-$ & $16 / 3$ & $18 / 9$ & $20 /-$ \\
\hline - & - & - & - & $45 /-$ & $42 / 6$ & $42 / 6$ & $55 /-$ & $55 /-$ & $60 /-$ & $72 / 6$ & - \\
\hline $12^{d}$ & $12^{\mathrm{d}}$ & $12^{d}$ & $12^{d}$ & $12^{d}$ & $12^{d}$ & $12^{d}$ & $12^{d}$ & $12^{d}$ & $12^{d}$ & $12^{d}$ & $10^{d}$ \\
\hline $3 /-$ & $3 /-$ & $3 /-$ & $3 /-$ & $3 /-$ & $3 /-$ & $3 /-$ & $3 /-$ & $3 /-$ & $3 /-$ & $3 /-$ & $3 /-$ \\
\hline $70 /-$ & $70 /-$ & $70 /-$ & $150 /-$ & $180 /$ - & $87 / 6$ & $65 /-$ & $60 /-$ & $60 /-$ & $60 /-$ & $55 /-$ & $60 /-$ \\
\hline - & - & - & - & $2 / 41 / 2$ & $2 / 11 / 2$ & $2 /-$ & $1 / 10$ & $22^{d}$ & $22^{\mathrm{d}}$ & $20^{d}$ & - \\
\hline - & - & - & - & $£ 7$ & $130 /-$ & $130 /-$ & $110 /-$ & $£ 5$ & $£ 5$ & $£ 5$ & - \\
\hline - & - & - & - & - & $65 /-$ & $65 /-$ & $70 /-$ & $70 /-$ & $70 /-$ & $70 /-$ & - \\
\hline - & - & - & - & - & $71 / 2^{d}$ & $71 / 2 d$ & $9 d$ & $9 d$ & $9^{d}$ & $10^{d}$ & - \\
\hline - & - & - & - & - & $9 / 3$ & $9 / 3$ & $10 /-$ & $10 /-$ & $12 / 6$ & $12 / 6$ & - \\
\hline- & - & - & - & $8^{d}$ & $6 \% / 4 d$ & $6^{d}$ & $6^{\mathrm{d}}$ & $6^{d}$ & $6^{d}$ & $6^{d}$ & - \\
\hline
\end{tabular}

赵 
du porc, du beurre, du café, du bois de chauffage, du charbon, des chandelles, de la mélasise et de l'huile à lampe. Nous avons attribué une pondération égale à chacun de ces produits. L'indice simple que l'on en décante illustre un mode d'agrégation encore peu familier aux historiens. ${ }^{21}$ Le tableau $\mathrm{V}$ reproduit les prix bruts, le tableau VI, l'indice des prix à la consommation. De la même manière, nous avons tenté ailleurs d'élaborer un indice de revenus et de niveau de vie pour les paysans. ${ }^{22}$

Afin d'illustrer un peu mieux l'utilité sur le plan analytique de ces séries mensuelles, nous avons calculé le mouvement saisonnier qui se dégage de notre indice de prix à la consommation. Il existe en effet des techniques statistiques qui permettent de dissocier la composante saisonnière des séries chronologiques des autres éléments qui définissent la configuration des courbes. Nous avons construit cet indice du mouvement saisonnier à partir de la méthode des moyennes mobiles pondérées. ${ }^{23}$ L'indice du mouvement saisonnier est défini clairement au tableau VII. Le lecteur pourra aussi trouver utile de reproduire cet indice sur un graphique simple, aux fins d'interprétation.

Encore que bien imparfait, un tel indice du mouvement saisonnier pourra, une fois affiné, nous servir à "désaisonnaliser" les séries chronologiques afin de mieux découper les composantes tendancielles et cycliques. D'autre part, il est essentiel de "désaisonnaliser" less données au moment de faire certaines comparaisons de courte période. Il se peut en effet qu'une baisse de prix observée entre avril et septembre soit le symptôme d'une amorce de mouvement conjoncturel à la baisse;

21 Nous avons choisi les 10 produits en fonction de la consommation générale, définie à partir d'un grand nombre de sources quantitatives et qualitatives (cf. notre communication au Colloque de statistique). Bien sûr, une pondération égale ne correspond pas à la consommation effective. Nous avons aussi utilisé des pondérations différentes inspirées de sources contemporaines. Toutefois, même des modifications radicales dans ces pondérations ne font pas varier sensiblement l'indice des prix qui en résulte.

22 Voir notre communication au Colloque de statistique.

23 Pour les détails de la méthode statistique, voir Daniel B. Suits, Statistics: An Introduction to Quantitative Economic Research (Chicago, 1963), 215-220. 


\section{TABLEAU VI}

Indice mensuel des prix à la consommation (10 produits, pondération égale, avril $1805=100$ )

\begin{tabular}{|c|c|c|c|c|c|c|c|c|}
\hline $\begin{array}{l}\text { nvier } \\
\text { evrier } \\
\text { ars } \\
\text { vril } \\
\text { ai } \\
\text { iin } \\
\text { illlet } \\
\text { oût } \\
\text { uptembre } \\
\text { etobre } \\
\text { ovembre } \\
\text { écembre }\end{array}$ & $\begin{array}{r}\bar{z} \\
\overline{100.0} \\
\overline{99} .3 \\
103.6 \\
92.6 \\
90.3 \\
92.4 \\
93.3 \\
95.9\end{array}$ & $\begin{array}{l}96.9 \\
97.0 \\
97.3 \\
97.5 \\
98.2 \\
89.5 \\
88.6 \\
86.7 \\
88.9 \\
90.8 \\
89.8 \\
88.4\end{array}$ & $\begin{array}{l}89.8 \\
90.2 \\
89.3 \\
93.1 \\
\overline{92.1} \\
93.4 \\
95.6 \\
\overline{95.4} \\
94.1 \\
91.3\end{array}$ & $\begin{array}{r}96.1 \\
95.2 \\
97.2 \\
96.6 \\
91.3 \\
91.5 \\
87.1 \\
96.7 \\
94.7 \\
101.7 \\
99.9 \\
97.5\end{array}$ & $\begin{array}{r}101.8 \\
109.7 \\
111.5 \\
115.9 \\
104.5 \\
99.1 \\
99.2 \\
105.5 \\
106.0 \\
118.4 \\
117.0\end{array}$ & $\begin{array}{l}122.3 \\
121.9 \\
122.0 \\
119.8 \\
109.2 \\
106.0 \\
110.7 \\
119.2 \\
125.7 \\
127.7 \\
131.0\end{array}$ & $\begin{array}{l}\overline{129.6} \\
130.1 \\
122.0 \\
119.1 \\
= \\
\overline{-} \\
\overline{116.4} \\
120.0 \\
124.6 \\
129.4\end{array}$ & $\begin{array}{l}119.8 \\
118.4\end{array}$ \\
\hline
\end{tabular}

* Le prix du porc n'était pas disponible pour ces deux mois. Mais à cause de sa grande stabilité, nous avons interpolé ce prix.

\section{TABLEAU VII}

Indice du mouvement saisonnier extrait de l'indice des prix à la consommation présenté au tableau VI

$\begin{array}{lllllr}\text { Janvier } & 101.9 & \text { Mai } & 98.5 & \text { Septembre } & 97.9 \\ \text { Février } & 103.3 & \text { Juin } & 94.3 & \text { Octobre } & 102.3 \\ \text { Mars } & 103.6 & \text { Juillet } & 94.3 & \text { Novembre } & 102.2 \\ \text { Avril } & 103.9 & \text { Août } & 98.5 & \text { Décembre } & 99.8\end{array}$

mais il se peut aussi qu'il ne s'agisse que d'un mouvement saisonnier et qu'en fait, une fois la composante saisonnière extraite, les prix montrent une légère hausse. Enfin, surtout pour une période où il faut souvent se contenter de séries partielles, ${ }^{24}$ un bon indice du mouvement saisonnier pourra permettre d'utiliser ces séries incomplètes après les avoir plus ou moins homogénéisées.

24 Souvent nous ne disposons que de données pour quelques mois répartis sur les quatre saisons. Il faut alors s'assurer qu'on n'introduit pas de biais en calculant une moyenne annuelle simple. Un bon indice saisonnier permettrait de dissocier ces prix de leur composante saisonnière. Ce problème n'a pas été clairement posé par Hamelin et Ouellet ("Le mouvement des prix agricoles...", in C. Galarneau et E. Lavoie, éd., op.cit., 37-39), encore qu'il soit moins important pour les comparaisons de longue période qui les intéressent. 


\section{III — En guise de modele}

Les données complémentaires et nouvelles incluses dans les deux premières sections, ne constituent en fait que des sousproduits de notre étude plus vaste sur la période 1790-1812. Elles prennent leur sens dans un modèle à trois paliers que nous avons conçu comme cutil de travail. Pour fin d'analyse, en effet, nous avons découpé l'économie du Bas-Canada en trois secteurs: le commerce international, l'économie domestique privée et l'économie publique. Dans chaque secteur, nous avons mis à jour de nouvelles données, puis amorcé des analyses qui révèlent certains mécanismes et certaines relations économiques. Nous renvoyons les lecteurs de la Revue à notre communication de juin pour l'ensemble des résultats préliminaires.

Cependant, l'on ne saurait s'enfermer dans le seul domaine économique. Nous souscrivons entièrement au postulat de Labrousse sur les rapports entre l'économique, le social et le mental, ${ }^{25}$ voire avec le politique: d'où notre participation à d'autres travaux sur les idéologies au tournant du XIX siècle et en démographie historique. ${ }^{26}$ Tous ces efforts veulent converger vers une approche mieux définie et plus formelle - donc davantage dénuée d'approximations ou d'ambiguités et propice aux discussions fructueuses - à cette sous-période très importante de restructuration économique et de luttes politiques, sociales et ethniques. Dans une approche ainsi élargie et clarifiée, une meilleure compréhension des mécanismes économiques devrait non seulement déboucher sur une étude d'économie historique, mais encore s'imbriquer avec d'autres éléments dans une histoire vraiment globale à la Braudel: une histoire mieux structurée, plus explicite dans ses postulats, au carrefour des diverses sciences de l'homme, tentant de reconstituer l'évolution de la

25 Ernest Labrousse, op.cit., 5.

26 Les deux auteurs ainsi que des historiens des universités de Montréal et d'Ottawa font partie d'un Groupe de recherche sur les idéologies dans la société canadienne-française. En outre, tous deux participent à un projet collectif de démographie historique (voir Hubert Charbonneau, et al., "La Démographie historique au Canada", Recherches sociographiques, 8 (mai-août 1967) : 214-217). Enfin, Jean-Pierre Wallot prépare une série d'études sur la période 1792-1812. 
société du Bas-Canada dans le contrepoint dialectique de l'ethnique, du politique, de l'économique, du social et du mental.

Il serait prématuré de tirer des conclusions audacieuses de résultats préliminaires: nos recherches se poursuivent. Toutefois, il est possible dès maintenant de prévoir qu'il faudra nuancer quelque peu la fresque originale de Ouellet, même sur le plan strictement économique. ${ }^{27}$ Il ne semble plus possible d'affirmer catégoriquement qu'en ce qui a trait à la première décennie du XIX $\mathrm{X}^{\mathrm{e}}$ siècle, "l'explication ultime de la diminution des exportations de céréales réside donc dans une défaillance de la production locale" ${ }^{28}$. Bref, la "crise agricole" avant 1812 nous apparaît mal établie. Elle est aussi une simplification de la réalité. Certaines analyses économiques préliminaires ${ }^{29}$ et une documentation qualitative abondante nous font entrevoir une réalité beaucoup plus complexe où se conjuguent une multitude de facteurs: instabilité d'une demande extérieure très erratique, accroissement notable de la main-d'œuvre non agricole (commerce du bois, construction de fortifications, etc.), variations régionales, fluctuations normales dans les récoltes, etc. Un indice sommaire des revenus agricoles rend même possible l'hypothèse d'une hausse du niveau de vie des paysans, particulièrement après $1805 .^{30}$

Mais notre propos n'est pas de chicaner Ouellet sur des détails (i.e. sur des aspects d'une courte période), encore que ceux-ci, à certains moments, affectent plus ou moins son interprétation "globale" de l'histoire du Bas-Canada dans les années qui nous intéressent ici. Le grand mérite de son Histoire éco-

27 Notre présent article ne s'attache qu'à ce plan.

28 F. Ouellet, Histoire économique et sociale..., 184; aussi pp. 171, 174-175, 180-188. L'auteur avait avancé cette opinion dès 1962 avec Jean Hamelin dans "La Crise agricole dans le Bas-Canada, 1802-37", Etudes Rurales (octobre-décembre 1962). Tous deux ont repris cette explication dans "Le Mouvement des prix agricoles...", in C. Galarneau et E. Lavoie, éd., op.cit., 40; aussi dans "Les Rendements agricoles dans les seigneuries et les cantons du Québec: 1700-1850", in ibid., 94, 103.

29 Gilles Paquet et Jean-Pierre Wallot, op. cit., 35-38.

30 Pour cet indice, cf. ibid., 29-32, graphiques 9 et 10. 
nomique et sociale, c'est d'avoir mis en place pour la première fois un cadre de référence socio-économique utilisable et fécond. Ouellet et Hamelin ont stimulé la recherche dans un secteur neuf: de cela, l'historiographie canadienne leur sera toujours redevable. Notre effort consiste à approfondir, préciser, affiner, rendre plus rigoureux et, si possible, améliorer ce cadre de référence par une plongée plus profonde dans une période plus courte. En ce sens, Ouellet, qui concevait son livre comme "un tremplin pour de futures conquêtes", commence déjà à atteindre son but. Nous souhaitons donc que plus de chercheurs explorent d'autres périodess, de sorte qu'éventuellement, nous disposions d'une véritable "histoire globale" de 1760 jusqu'au $\mathrm{XX}^{\mathrm{e}}$ siècle (et pourquoi pas avant 1760 ?).

Gilles PaQUeT

Université Carleton

JEAN-PIERRE WALLOT

Musée national 